\title{
Status of Absolute Neutrino Mass and Double Beta Decay
}

\author{
H.V. Klapdor-Kleingrothaus* ${ }^{* \dagger}$ \\ Max-Planck-Institut für Kernphysik \\ P.O. Box 1039 80, D-69029 Heidelberg, Germany \\ E-mail: H. Kl $\mathrm{L}$ apdor@mpi-hd.mpg.de
}

\begin{abstract}
The first evidence for neutrinoless double beta decay has been observed in the HEIDELBERG-MOSCOW experiment, which is the most sensitive double beta decay experiment since ten years. This is the first evidence for lepton number violation and proves that the neutrino is a Majorana particle. It further shows that neutrino masses are degenerate. In addition it puts several stringent constraints on other physics beyond the Standard Model. In particular it opens the door to test various supersymmetric theory scenarios, for example it gives the sharpest limit on the parameter $\lambda_{111}^{\prime}$ in the R-parity violating part of the superpotential, and gives information on the splitting of the sneutrino-antisneutrino system. The result from the HEIDELBERG-MOSCOW experiment is consistent with recent results from CMB investigations, with high energy cosmic rays, with the result from the g-2 experiment and with recent theoretical work. It is indirectly supported by the analysis of other Ge double beta experiments. Recent criticism of various kind has been shown to be wrong, among others by measurements with a ${ }^{214} B i$ source $\left({ }^{226} R a\right)$, by simulation of the background in the range of $\mathrm{Q}_{\beta \beta}$ by GEANT4, and by deeper investigation of statistical features such as sensitivity of peak search, and relevance of width of window of analysis.

A Test Facility for the GENIUS project has come into operation on May 5, 2003. This is the first time that this novel technique for extreme background reduction in search for rare decays is applied under the background conditions of an underground laboratory.
\end{abstract}

\section{Introduction}

Double beta decay is the most sensitive probe to test lepton number conservation. Further it seems to be the only way to decide about the Dirac or Majorana nature of the neutrino.

${ }^{*}$ Speaker.

${ }^{\dagger}$ Spokesman of Heidelberg-Moscow and GENIUS Collaborations (http://www.mpi-hd.mpg.de.non_acc/). 
Double beta decay can contribute decisively to the field of neutrino physics also by setting an absolute scale to neutrino masses, which cannot be observed from neutrino oscillation experiments.

The observable of double beta decay is the effective neutrino mass

$$
\langle m\rangle=\left|\sum U_{e i}^{2} m_{i}\right|=\left|m_{e e}^{(1)}\right|+e^{i \phi_{2}}\left|m_{e e}^{(2)}\right|+e^{i \phi_{3}}\left|m_{e e}^{(3)}\right|,
$$

with $U_{e i}$ denoting elements of the neutrino mixing matrix, $m_{i}$ neutrino mass eigenstates, and $\phi_{i}$ relative Majorana $\mathrm{CP}$ phases. It can be written in terms of oscillation parameters $[1 \overline{1} \overline{1}$

$$
\begin{aligned}
\left|m_{e e}^{(1)}\right| & =\left|U_{e 1}\right|^{2} m_{1}, \\
\left|m_{e e}^{(2)}\right| & =\left|U_{e 2}\right|^{2} \sqrt{\Delta m_{21}^{2}+m_{1}^{2}}, \\
\left|m_{e e}^{(3)}\right| & =\left|U_{e 3}\right|^{2} \sqrt{\Delta m_{32}^{2}+\Delta m_{21}^{2}+m_{1}^{2}} .
\end{aligned}
$$

The effective mass $\langle m\rangle$ is related with the half-life for $0 \nu \beta \beta$ decay via $\left(T_{1 / 2}^{0 \nu}\right)^{-1} \sim$ $\left\langle m_{\nu}\right\rangle^{2}$, and for the limit on $T_{1 / 2}^{0 \nu}$ deducible in an experiment we have

$$
T_{1 / 2}^{0 \nu} \sim \epsilon \times a \sqrt{\frac{M t}{\Delta E B}},
$$

Here $a$ is the isotopical abundance of the $\beta \beta$ emitter; $M$ is the active detector mass; $t$ is the measuring time; $\Delta E$ is the energy resolution; $B$ is the background count rate and $\epsilon$ is the efficiency for detecting a $\beta \beta$ signal. Determination of the effective mass fixes the

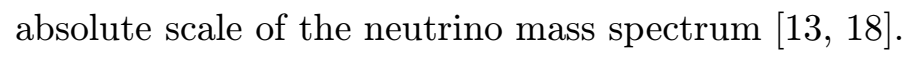

In this paper we will describe in section II the recent evidence for neutrinoless double

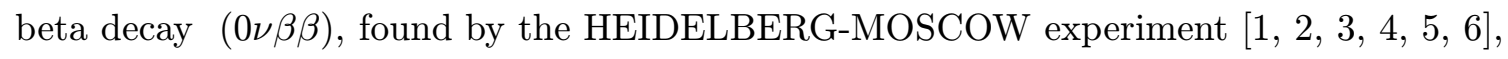
which is since ten years now the most sensitive double beta experiment worldwide.

This result is

$$
\mathrm{T}_{1 / 2}^{0 \nu}=(0.8-18.3) \times 10^{25} \mathrm{y} \quad(95 \% \text { c.l. })
$$

with best value of $\mathrm{T}_{1 / 2}^{0 \nu}=1.5 \times 10^{25} \mathrm{y}$. Double beta decay is the slowest nuclear decay process observed until now in nature. Assuming the neutrino mass mechanism to dominate the decay amplitude, we can deduce

$$
\left\langle m_{\nu}\right\rangle=(0.11-0.56) \mathrm{eV} \quad(95 \% \text { c.l. })
$$

This value we obtain using the nuclear matrix element of [를 $\left.\bar{B}_{1}\right]$. Allowing for an uncertainty

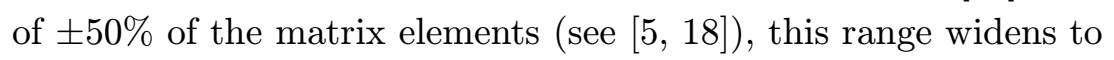

$$
\left\langle m_{\nu}\right\rangle=(0.05-0.84) \mathrm{eV}
$$

The result (2) and (3) determines the neutrino mass scenario to be degenerate [1 The common mass eigenvalue follows then to be

$$
m_{\text {com }}=(0.05-3.2) \mathrm{eV} \quad(95 \%)
$$




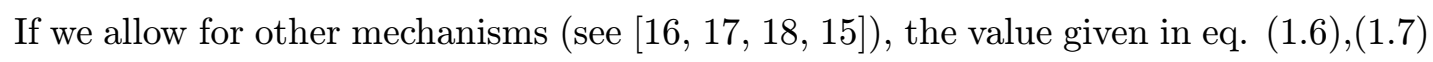
has to be considered as an upper limit. In that case very stringent limits arise for many other fields of beyond standard model physics. To give an example, it gives the sharpest limit on the Yukawa coupling $\lambda_{111}^{\prime}$ in the R-parity violating part of the superpotential [20 $\left.2 \overline{2}\right]$. It also gives information on R-parity conserving supersymmetry. New R-parity conserving SUSY contributions to $0 \nu \beta \beta$ decay occur at the level of box diagrams [2] $\left[\begin{array}{l}\overline{1} \\ 1\end{array}\right]$. Double beta decay then yields information on the mass splitting in the sneutrino-antisneutrino system

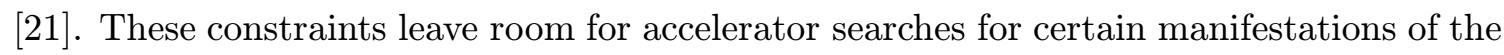
second and third generation (B-L)-violating sneutrino mass term, but are most probably too tight for first generation (B-L)-violating sneutrino masses to be searched for directly. It has been discussed recently [i] $\left.{ }_{1}^{2} \overline{2}\right]$ that $0 \nu \beta \beta$ decay by R-parity violating SUSY experimentally may not be excluded, although this would require making $\mathrm{R}$-parity violating couplings generation dependent.

We show, in section III that indirect support for the observed evidence for neutrinoless double beta decay evidence comes from analysis of other Ge double beta experiments (though they are by far less sensitive, they yield independent information on the background in the region of the expected signal).

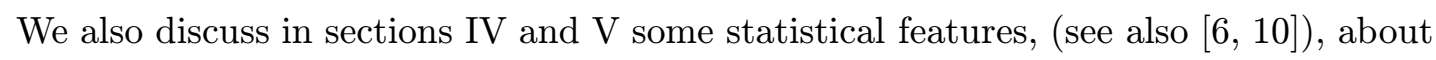
which still wrong ideas are around, as well as background simulations with the program GEANT4 [i] 2 2], which disprove some recent criticism, and finally the results of measurements with a ${ }^{214} B i$ source [i] $\left.{ }_{1}^{1}\right]$ which show that the criticism given by C.E. Aalseth et al. (see Mod. Phys. Lett. A17 (2002) 1475-1478) is wrong (see also section VI).

In section VII we give a short discussion, stressing that the evidence for neutrinoless

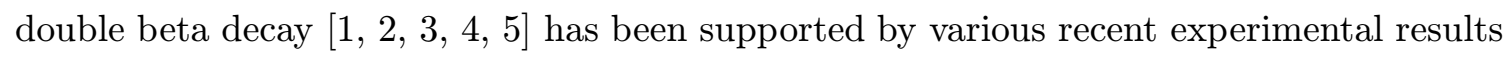
from other fields of research (see Table $\left.\underline{1}_{1}^{1}\right)$. It is consistent [1] $\left.{ }_{-1}^{1} \bar{g}\right]$ with recent results from

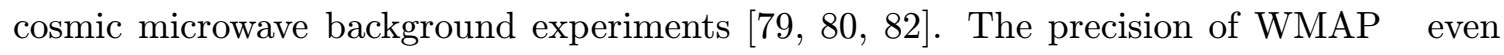
allows to rule out some old-fashioned nuclear double beta decay matrix elements (see [8in]

It has been shown to be consistent with the neutrino masses required for the Z-burst

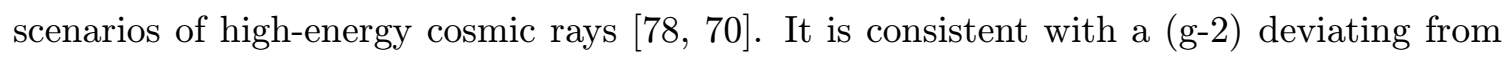
the standard model expectation [i] $\left.\left.{ }_{1}^{1} \overline{1}\right]\right]$. It is consistent also with the limit from the tritium decay experiments [i50 $[1]$ but the allowed $95 \%$ confidence range still extends down to a range which cannot be covered by future tritium experiments. It is further supported by recent

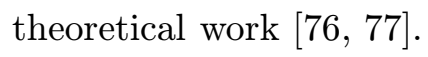

Cosmological experiments like WMAP are now on the level that they can seriously contribute to terrestrial research. The fact that WMAP and less strictly also the tritium experiments cut away the upper part of the allowed range for the degenerate neutrino mass

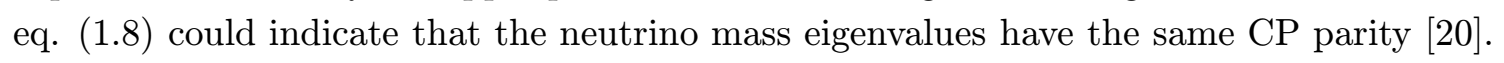

Finally we briefly comment in section VIII about the possible future of the field of double beta decay. First results from GENIUS-TF which has come into operation on May 5, 2003 in Gran Sasso with first in world $10 \mathrm{~kg}$ of naked Germanium detectors in liquid

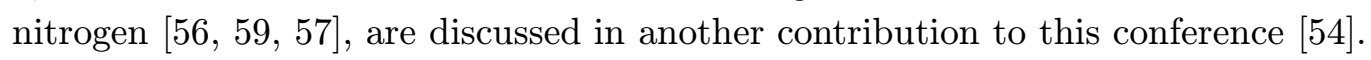




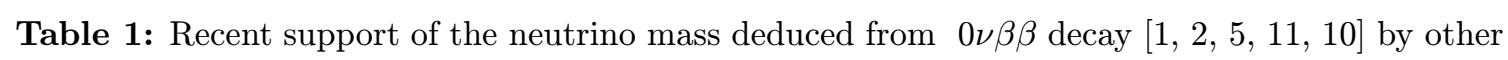
experiments, and by theoretical work.

\begin{tabular}{|c|c|c|}
\hline Experiment & References & $m_{\nu}($ degenerate $\nu$ 's $)(\mathrm{eV})$ \\
\hline $0 \nu \beta \beta$ & 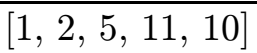 & $0.05-3.2$ \\
\hline WMAP & 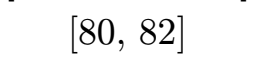 & $<0.23$, or 0.33, or 0.50 \\
\hline $\mathrm{CMB}$ & {$[\overline{\overline{9}} \overline{\overline{9}}]$} & $<0.7$ \\
\hline CMB+LSS+X-ray gal. Clust. & 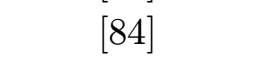 & $\sim 0.2 \mathrm{eV}$ \\
\hline $\mathrm{Z}-$ burst & {$[170,1,78]$} & $0.08-1.3$ \\
\hline g-2 & {$\left[\begin{array}{ll}17 \\
1\end{array}\right]$} & $>0.2$ \\
\hline Tritium & {$[6 \overline{1}]$} & $<2.2-2.8$ \\
\hline$\nu$ oscillation & 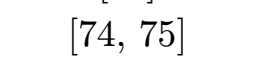 & $>0.04$ \\
\hline \multicolumn{3}{|l|}{ Theory: } \\
\hline $\begin{array}{c}\mathrm{A}_{4} \text {-symmetry } \\
\text { identical quark }\end{array}$ & 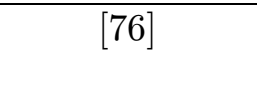 & $>0.2$ \\
\hline and $\nu$ mixing at GUT scale & {$\left[\begin{array}{l}\overline{7} \\
\mathbf{Z} \overline{7}\end{array}\right]$} & $>0.1$ \\
\hline $\begin{array}{l}\text { Alternative cosmological } \\
\text { 'concordance model' }\end{array}$ & {$[\overline{8} \overline{5}]$} & order of eV \\
\hline
\end{tabular}

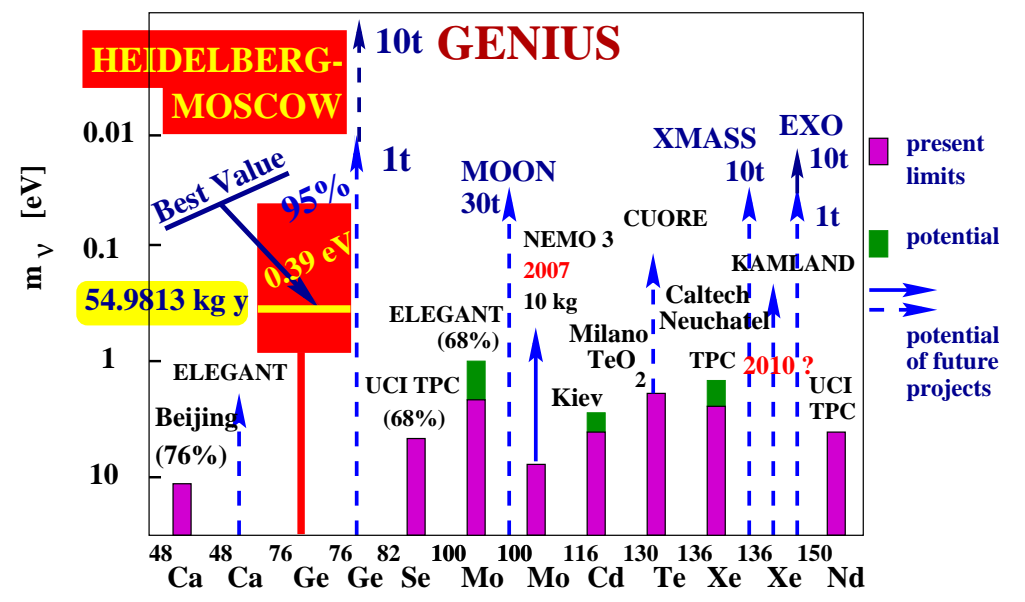

Figure 1: Present sensitivity, and expectation for the future, of the most promising $\beta \beta$ experiments. Given are limits for $\langle m\rangle$, except for the HEIDELBERG-MOSCOW experiment where the recently observed value is given ( $95 \%$ c.l. range and best value). Framed parts of the bars: present status; not framed parts: future expectation for running experiments; solid and dashed lines: experiments

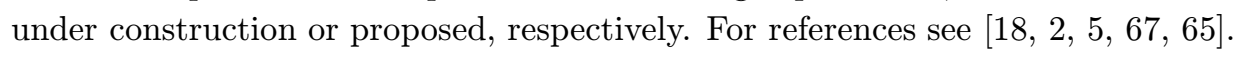

\section{Evidence for the neutrinoless decay mode}

The status of present double beta experiments is shown in Fig. $\prod_{1}^{1}$ and is extensively discussed in [i] $[\overline{8} \overline{1}]$. The HEIDELBERG-MOSCOW experiment using the largest source strength of 11 $\mathrm{kg}$ of enriched ${ }^{76} \mathrm{Ge}$ (enrichment $86 \%$ ) in form of five HP Ge-detectors is running since

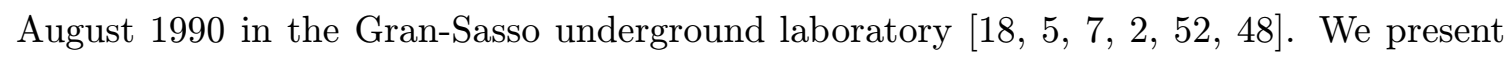
here the data until May 2000, an analysis of three more years data, until May 20, 2003, 
will be given shortly.

The data taken in the period $\mathrm{Au}$ gust 1990-May 2000 (54.9813 kg y, or 723.44 mol-years) are shown in Fig. in the section around the $\mathrm{Q}_{\beta \beta}$ value

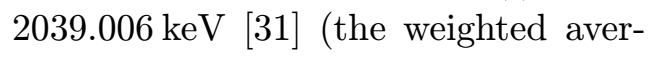
age of the four measurements given in

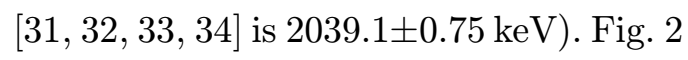
is identical with Fig. 嗃 in that we show here the original energy binning of the data of $0.36 \mathrm{keV}$. These

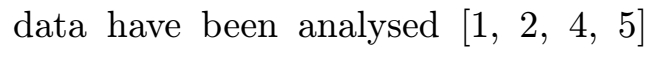
with various statistical methods, with the Maximum Likelihood Method and with the Bayesian method (see [剞).

Our peak search procedure (for de-

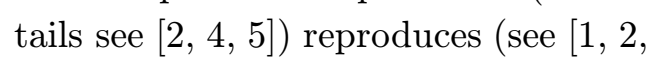
售, 它) $\gamma$-lines at the positions of known

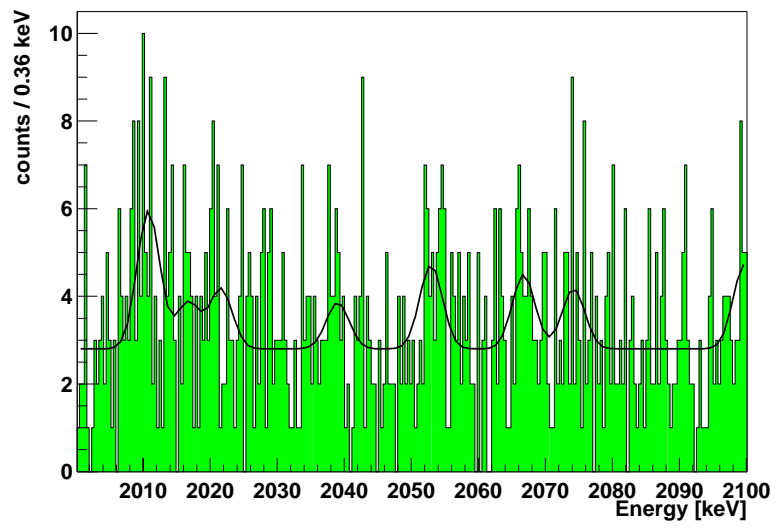

Figure 2: The spectrum taken with the ${ }^{76} \mathrm{Ge}$ detectors Nr. 1,2,3,4,5 over the period August 1990 - May $2000(54.9813 \mathrm{~kg}$ y) in the original $0.36 \mathrm{keV}$ binning, in the energy range $2000-2100 \mathrm{keV}$. Simultaneous fit of the ${ }^{214} \mathrm{Bi}$ lines and the two high-energy lines yield a probability for a line at $2039.0 \mathrm{keV}$ of $91 \%$. weak lines [2] $\left.{ }^{2} \bar{g}_{1}\right]$ from the decay of ${ }^{214} \mathrm{Bi}$

at 2010.7, 2016.7, 2021.8 and $2052.9 \mathrm{keV}$. In addition, a line centered at $2039 \mathrm{keV}$ shows

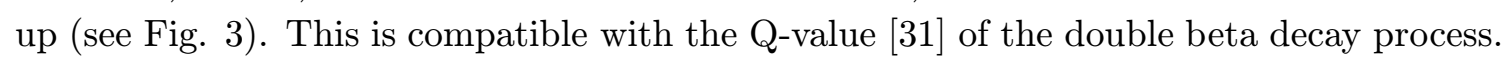
The Bayesian analysis yields, when analysing a $\pm 5 \sigma$ range around $\mathrm{Q}_{\beta \beta}$ (which is the usual procedure when searching for resonances in high-energy physics) a confidence level (i.e. the probability K) for a line to exist at $2039.0 \mathrm{keV}$ of $96.5 \%$ c.l. $(2.1 \sigma)$ (see Fig. $\underline{\underline{p}_{1}}$ ). We repeated the analysis for the same data, but except detector 4 , which had no muon shield and a slightly worse energy resolution $(46.502 \mathrm{~kg}$ y). The probability we find for a line at

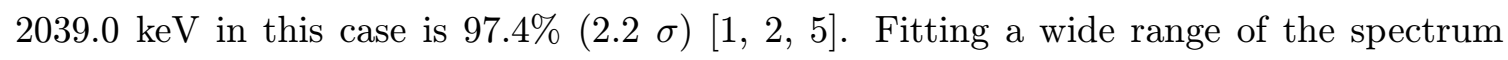
yields a line at $2039 \mathrm{keV}$ at $91 \%$ c.l. (see Fig.2.
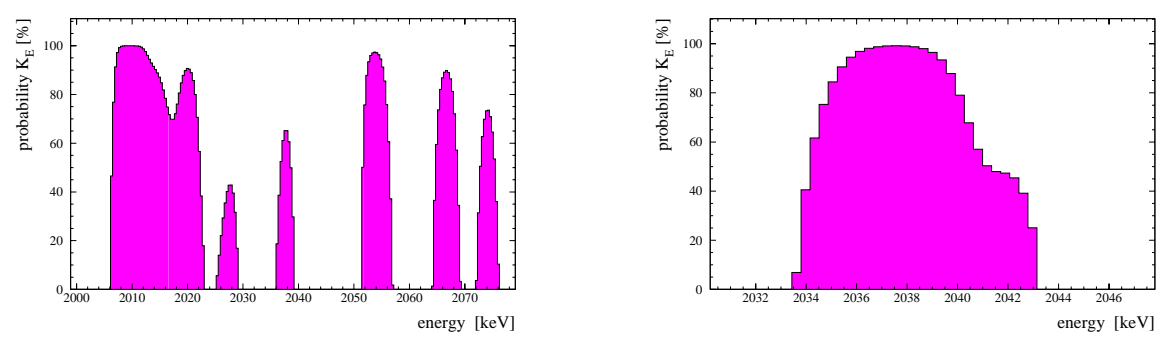

Figure 3: Left: Probability $\mathrm{K}$ that a line exists at a given energy in the range of 2000-2080 keV derived via Bayesian inference from the spectrum shown in Fig. $\overline{2}_{\mathbf{r}}^{-1}$ Right: Result of a Bayesian scan for lines as in the left part of this figure, but in an energy range of $\pm 5 \sigma$ around $\mathrm{Q}_{\beta \beta}$.

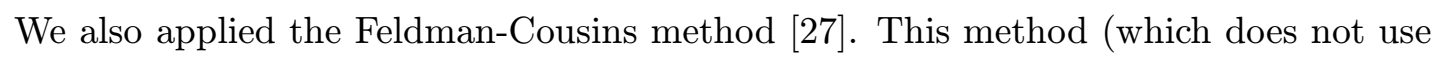
the information that the line is Gaussian) finds a line at $2039 \mathrm{keV}$ on a confidence level of 
$3.1 \sigma(99.8 \%$ c.l. $)$.

In addition to the line at $2039 \mathrm{keV}$ we find candidates for lines at energies beyond $2060 \mathrm{keV}$ and around $2030 \mathrm{keV}$, which at present cannot be attributed. This is a task of nuclear spectroscopy.

Important further information can be obtained from the time structures of the individual events. Double beta events should behave as single site events i.e. clearly different from a multiple scattered $\gamma$-event. We have tried to differentiate between these different types of events by pulse shape analysis. For this purpose, we have developed three methods of

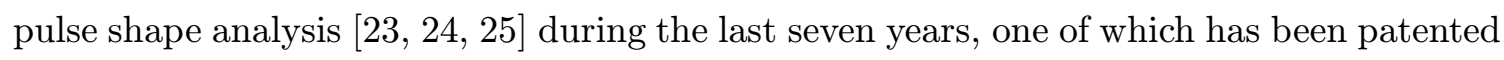
and therefore only published recently.

Installation of Pulse Shape Analysis (PSA) has been performed in 1995 for the four large detectors. They are running since November 1995 with PSA. The measuring time with PSA from November 1995 until May 2000 is $36.532 \mathrm{~kg}$ years, for detectors 2,3,5 it is $28.053 \mathrm{~kg}$ y.

With proper normalization concerning the running times ( $\mathrm{kg} y$ ) of the full and the SSE spectra, and the efficiency of pulse shape identification, we see that almost the full signal remains after the single site cut (best value), while normal $\gamma$-lines (consisting to $\sim 80 \%$ of multiple site events and only to $\sim 20 \%$ of single events) are correspondingly reduced.

The possibility, that the single site signal is the double escape line corresponding to a (much more intense!) full energy peak of a $\gamma$-line, at $2039+1022=3061 \mathrm{keV}$ is excluded from the high-energy part of our spectrum.

A careful simulation of the different components of radioactive background in the HEIDELBERG-MOSCOW experiment has been performed recently by a new Monte Carlo

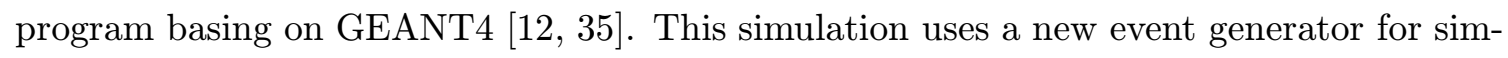
ulation of radioactive decays basing on ENSDF-data and describes the decay of arbitrary radioactive isotopes including $\alpha, \beta$ and $\gamma$ emission as well as conversion electrons and $\mathrm{X}$ ray emission. Also included in the simulation is the influence of neutrons in the energy range from thermal to high energies up to $100 \mathrm{MeV}$ on the measured spectrum. Elastic and inelastic reactions, and capture have been considered, and the corresponding production of radioactive isotopes in the materials of the setup. The neutron fluxes and energy distributions were taken from published measurements performed in the Gran Sasso. Also simulated was the influence of the cosmic muon flux measured in the Gran Sasso, on the measured spectrum.

The simulation gives no indication that the signal at $2039 \mathrm{keV}$ comes from a known background line. In particular, the simulation shows, that e.g. decays of ${ }^{77} G e,{ }^{76} G a$ or ${ }^{228} A c$, should not lead to signals visible in our measured spectra near the signal at $\mathrm{Q}_{\beta \beta}$. For details we refer to [i] $\left.{ }_{2}^{2} \overline{1}\right]$.

\section{Support of Evidence From Other Ge-Experiments and From Recent Measurements With a ${ }^{214} B i$ Source}

It has been mentioned in Section II, that by the peak search procedure developped [20, on basis of the Bayes and Maximum Likelihood Methods, exploiting as important input 
parameters the experimental knowledge on the shape and width of lines in the spectrum, weak lines of ${ }^{214} \mathrm{Bi}$ have been identified at the energies of 2010.78, 2016.7, 2021.6 and

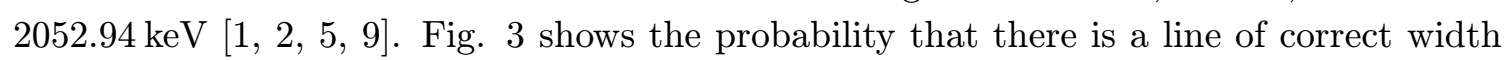
and of Gaussian shape at a given energy, assuming all the rest of the spectrum as flat background (which is a highly conservative assumption).

The intensities of these ${ }^{214} \mathrm{Bi}$ lines have been shown to be consistent with other, strong $\mathrm{Bi}$ lines in the measured spectrum according to the branching ratios given in the Table of Isotopes [2 $[2 \overline{9}]$, and to Monte Carlo simulation of the experimental setup [i5i]. Note that the $2016 \mathrm{keV}$ line, as an E0 transition, can be seen only by coincident summing of the two successive lines $E=1407.98 \mathrm{keV}$ and $E=609.316 \mathrm{keV}$. Its observation proves that the ${ }^{238} \mathrm{U}$ impurity from which it is originating, is located in the $\mathrm{Cu}$ cap of the detectors. Recent measurements of the spectrum of a ${ }^{214} \mathrm{Bi}$ source as function of distance source-detector confirm this interpretation [i] 1 in].

Premature estimates of the $\mathrm{Bi}$ intensities given in Aalseth et.al, hep-ex/0202018 and Feruglio et al., Nucl. Phys. B 637 (2002), 345, thus are incorrect, because this long-known

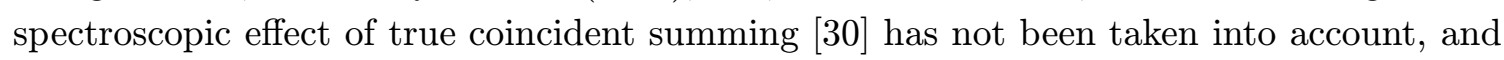

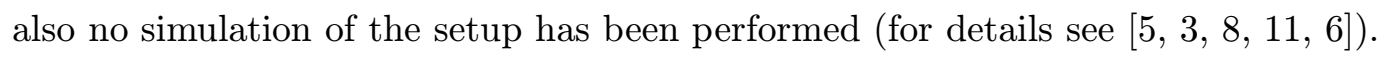

These ${ }^{214} \mathrm{Bi}$ lines occur also in other investigations of double beta decay of $\mathrm{Ge}$ - and even more important - also the additional structures in Fig. 2, which cannot be attributed

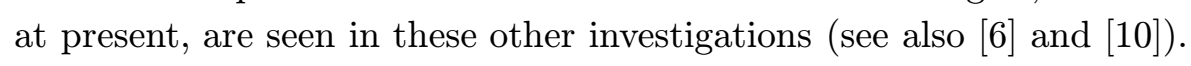
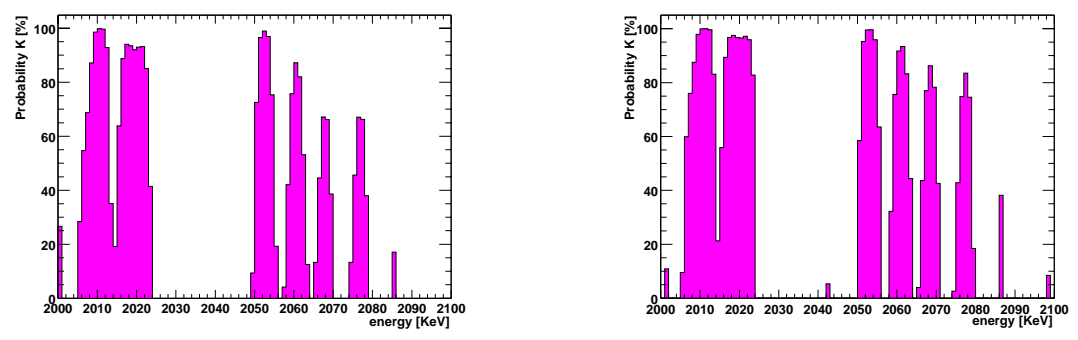

Figure 4: Result of the peak-search procedure performed for the UCBS/LBL spectrum [399] (left: Maximum Likelihood method, right: Bayes method). On the y axis the probability of having a line at the corresponding energy in the spectrum is shown.
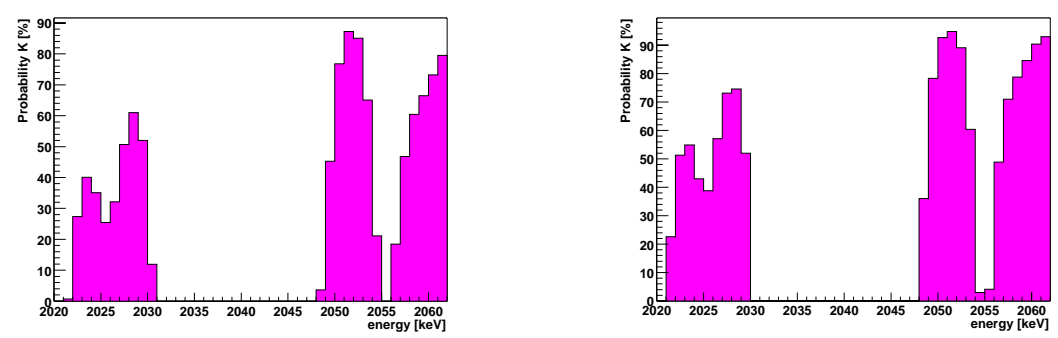

Figure 5: Result of the peak-search procedure performed for the ITEP/YePI spectrum [400"] 

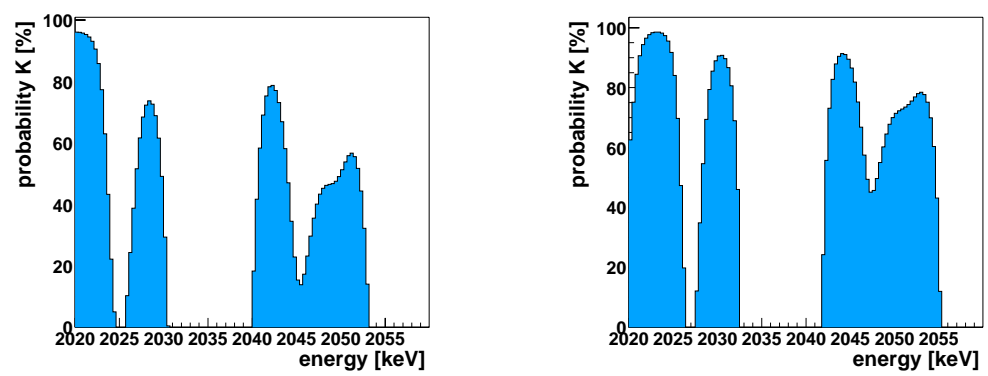

Figure 6: Result of the peak-search procedure performed for the IGEX spectrum [64]. Left: Maximum Likelihood method, right: Bayes method. On the y axis the probability of having a line at the corresponding energy in the specrtum is shown.

There are three other Ge experiments which have looked for double beta decay of ${ }^{76} \mathrm{Ge}$.

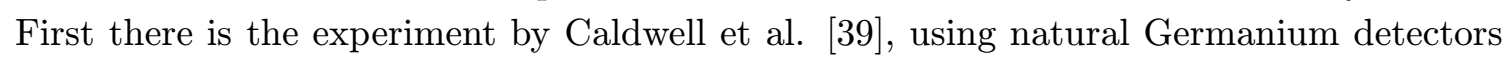
(7.8\% abundance of ${ }^{76} \mathrm{Ge}$, compared to $86 \%$ in the HEIDELBERG-MOSCOW experiment). This was the most sensitive natural Ge experiment. With their background a factor of 9 higher than in the HEIDELBERG-MOSCOW experiment and their measuring time of $22.6 \mathrm{~kg}$ years, they had a statistics of the background by a factor of almost four $\mathrm{l}$ a $\mathrm{r} \mathrm{g}$ e $\mathrm{r}$ than in the HEIDELBERG-MOSCOW experiment. This gives useful information on the composition of the background.

Applying the same method of peak search as used in Fig. '3i, yields

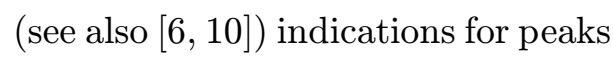
essentially at the same energies as

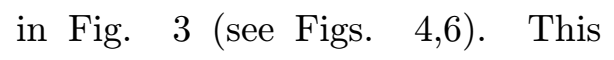
shows that these peaks are not fluctuations. In particular it sees the $2010.78,2016.7,2021.6$ and $2052.94 \mathrm{keV}$ ${ }^{214} \mathrm{Bi}$ lines, but a $1 \mathrm{~s}$ o the unattributed lines at higher energies. It finds, however, n o line at $2039 \mathrm{keV}$. This is consistent with the expectation from the rate found in the HEIDELBERG-MOSCOW experiment. About 16 observed events in the latter correspond to to 0.6 expected events in the Caldwell ex-

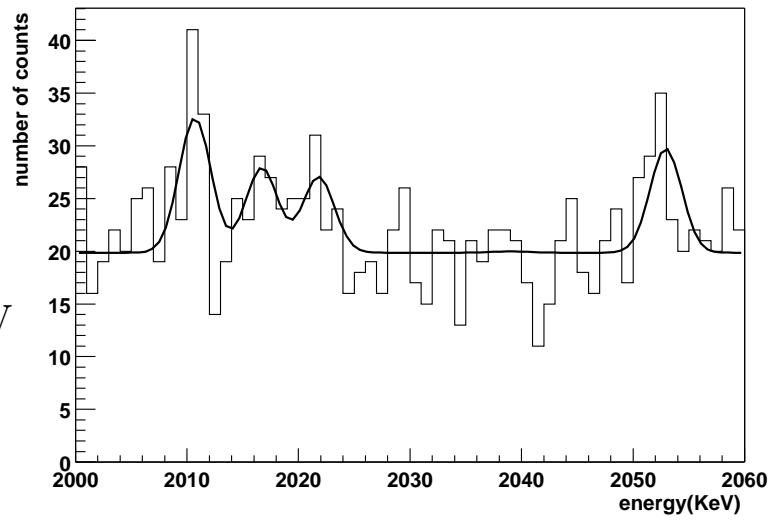

Figure 7: Analysis of the spectrum measured by D. Caldwell et al. [399], with the Maximum Likelihood Method, in the energy range $2000-2060 \mathrm{keV}$ assuming lines at $2010.78,2016.70,2021.60,2052.94,2039.0 \mathrm{keV}$. No indication for a signal at $2039 \mathrm{keV}$ is observed in this case. periment, because of the use of nonenriched material and the shorter measuring time. Fit of the Caldwell spectrum allowing

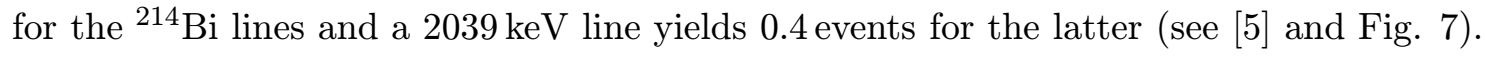

The first experiment using enriched (but not high-purity) Germanium 76 detectors

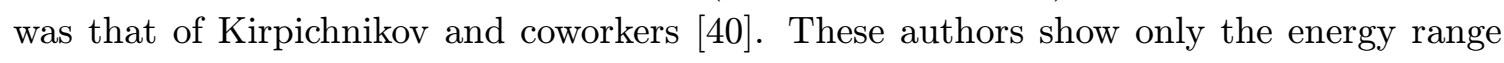


between 2020 and $2064 \mathrm{keV}$ of their measured spectrum. The peak search procedure finds also here indications of lines around $2028 \mathrm{keV}$ and $2052 \mathrm{keV}$ (see Fig. 产), but n o t any indication of a line at $2039 \mathrm{keV}$. This is consistent with the expectation, because for their low statistics of $2.95 \mathrm{~kg}$ y they would expect here (according to HEIDELBERG-MOSCOW) 0.9 counts.

Another experiment (IGEX) used between 6 and $8.8 \mathrm{~kg}$ of enriched ${ }^{76} \mathrm{Ge}$, but collected since beginning of the experiment in the early nineties till shutdown in 1999 only 8.8

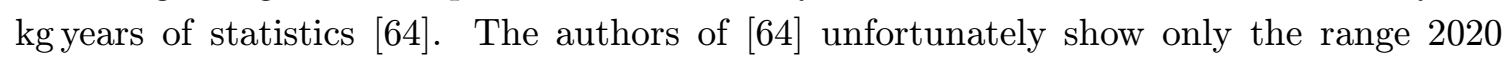
to $2060 \mathrm{keV}$ of their measured spectrum in detail. Fig. scanning of this range. Clear indications are seen for the lines at 2021 and $2052 \mathrm{keV}$, but also of the unidentified structure around $2030 \mathrm{keV}$. Because of the conservative assumption on the background treatment in the scanning procedure (see above) there is no chance to see a signal at $2039 \mathrm{keV}$ because of the 'hole' in the background of that spectrum (see Fig.

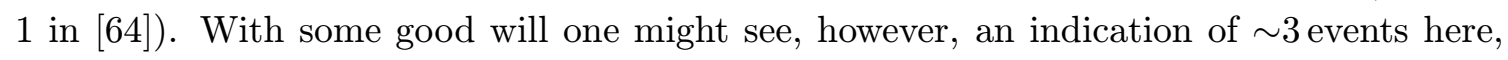
consistent with the expectation of the HEIDELBERG-MOSCOW experiment of 2.6 counts.

\section{Statistical Features:}

\section{Sensitivity of Peak Search, Analysis Window}

At this point it may be useful to demonstrate the potential of the used peak search procedure. Fig. $\bar{i}$ ishows a spectrum with Poisson-generated background of 4 events per channel and a Gaussian line with width (standard deviation) of 4 channels centered at channel 50 , with intensity of 10 (left) and 100 (right) events, respectively. Fig. 1밈, shows the result of the analysis of spectra of different line intensity with the Bayes method (here Bayes 1-4 correspond to different choice of the prior distribution: (1) $\mu(\eta)=1$ (flat), (2) $\mu(\eta)=1 / \eta$, (3) $\mu(\eta)=1 / \sqrt{\eta}$, (4) Jeffrey's prior) and the Maximum Likelihood Method. For each prior 1000 spectra have been generated with equal background and equal line intensity using

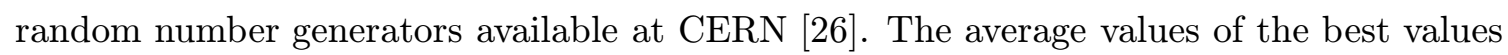
agree (see Fig. in Fig. $\bar{\beta}_{1}^{\prime \prime}$ left).

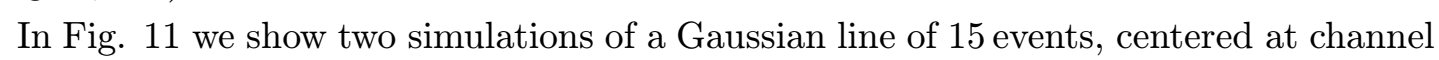
50, again with width (standard deviation) of 4 channels, on a Poisson-distributed background with 0.5 events/channel. The figure gives an indication of the possible degree of deviation of the energy of the peak maximum from the transition energy, on the level of statistics collected in experiments like the HEIDELBERG-MOSCOW experiment (here one channel corresponds to $0.36 \mathrm{keV}$ ). This should be considered when comparing Figs.

The influence of the choice of the energy range of the analysis around $Q_{\beta \beta}$ has been thoroughly discussed in [2]

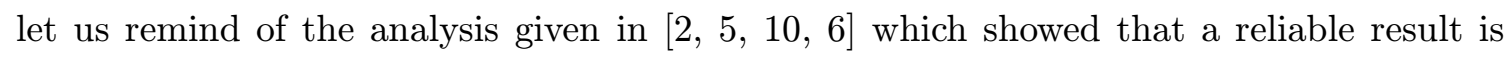
obtained for a range of analysis of not smaller than 35 channels (i.e. \pm 18 channels) one channel corresponding to $0.36 \mathrm{keV}$ in the HEIDELBERG-MOSCOW experiment (see Fig. (9i). This is an important result, since it is of course important to keep the range of analysis as s m a 11 as possible, to avoid to include lines in the vicinity of the weak 

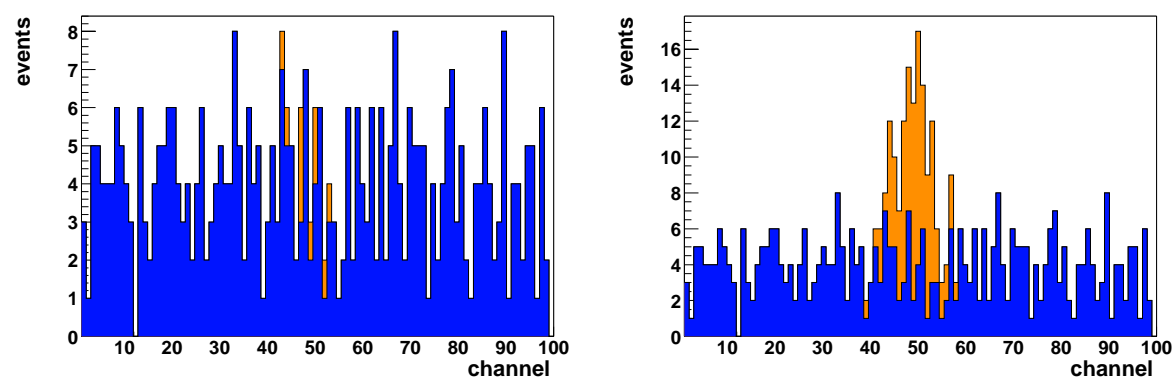

Figure 8: Example of a random-generated spectrum with a Poisson distributed background with 4.0 events per channel and a Gaussian line centered in channel 50 (line-width corresponds to a standard-deviation of $\sigma=4.0$ channels). The left picture shows a spectrum with a line-intensity of 10 events, the right spectrum a spectrum with a line-intensity of 100 events. The background is

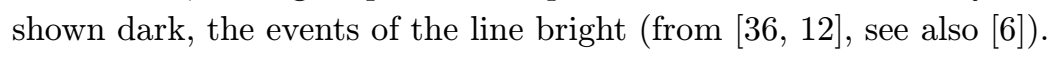

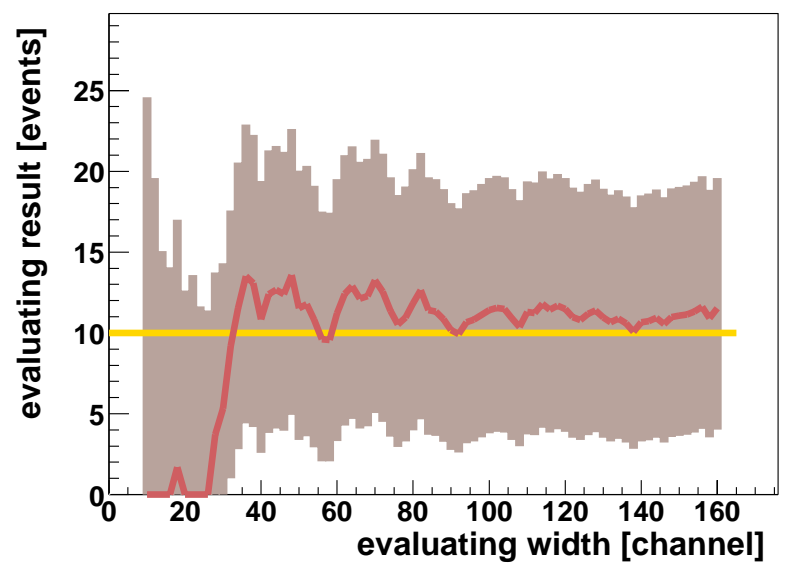

Figure 9: Result of an analysis as function of the evaluation width. The used spectrum consists of a Poisson distributed background with 4 events per channel, and a line of 10 events (see Fig. . left part). The dark area corresponds to a $68.3 \%$ confidence area with the dark line being the best value. Below an evaluation width of 35 channels the result becomes unreliable, above 35 channels the result is stable (see also $[\overline{6} \mid \bar{l}$ ).

signal into the background. This unavoidably occurs when e.g. proceeding as suggested in F. Feruglio et al., hep-ph/0201291 and Nucl. Phys. B 637 (2002) 345-377, Aalseth et. al., hep-ex/0202018 and Mod. Phys. Lett. A 17 (2002) 1475, Yu.G. Zdesenko et. al., Phys. Lett. B 546 (2002) 206, A. Ianni, in Press NIM 2004. The arguments given in those

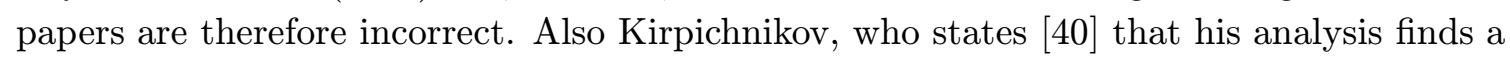
$2039 \mathrm{keV}$ signal in the HEIDELBERG-MOSCOW spectrum on a 4 sigma confidence level

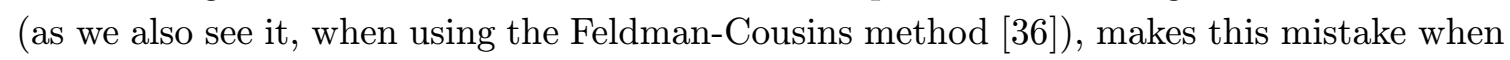
analyzing the pulse-shape spectrum.

\section{Simulation with GEANT4}

Finally the background around $Q_{\beta \beta}$ will be discussed from the side of simulation. A 
very careful new simulation of the different components of radioactive background in the HEIDELBERG-MOSCOW experiment has been performed recently by a new Monte Carlo

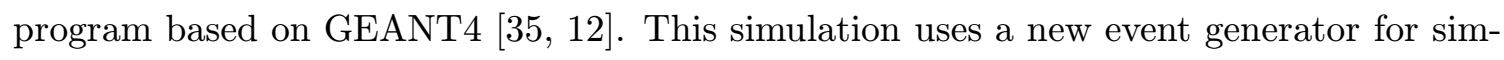
ulation of radioactive decays basing on ENSDF-data and describes the decay of arbitrary radioactive isotopes including alpha, beta and gamma emission as well as conversion electrons and X-ray emission. Also included in the simulation is the influence of neutrons in the energy range from thermal to high energies up to $100 \mathrm{MeV}$ on the measured spectrum. Elastic and inelastic reactions, and capture have been taken into account, and the corresponding production of radioactive isotopes in the setup. The neutron fluxes and energy distributions were taken from published measurements performed in the Gran Sasso.
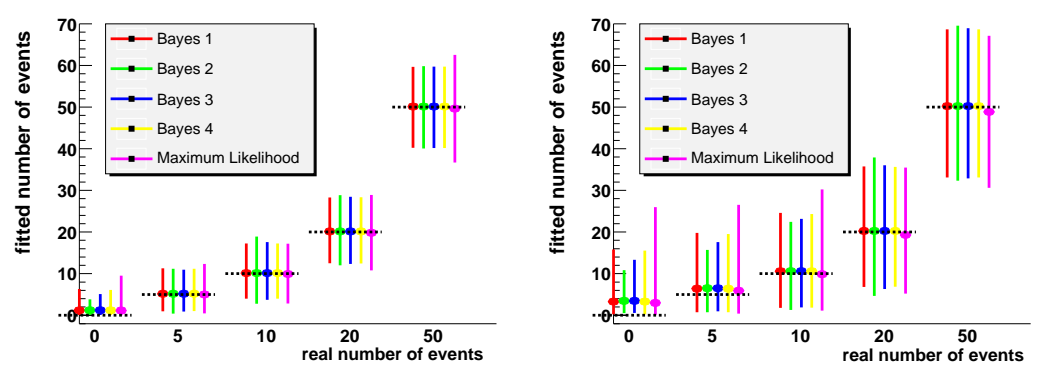

Figure 10: Results of analysis of random-number generated spectra, using Bayes and Maximum Likelihood method (the first one with different prior distributions). For each number of events in the simulated line, shown on the x-axis, 1000 random generated spectra were evaluated with the five given methods. The analysis on the left side was performed with an Poisson distributed background of 0.5 events per channel, the background for the spectra on the right side was 4.0 events per channel. Each vertical line shows the mean value of the calculated best values (thick points) with the $1 \sigma$ error area. The mean values are in good agreement with the expected values (horizontal

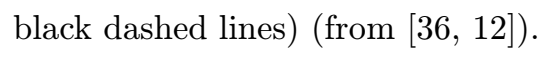
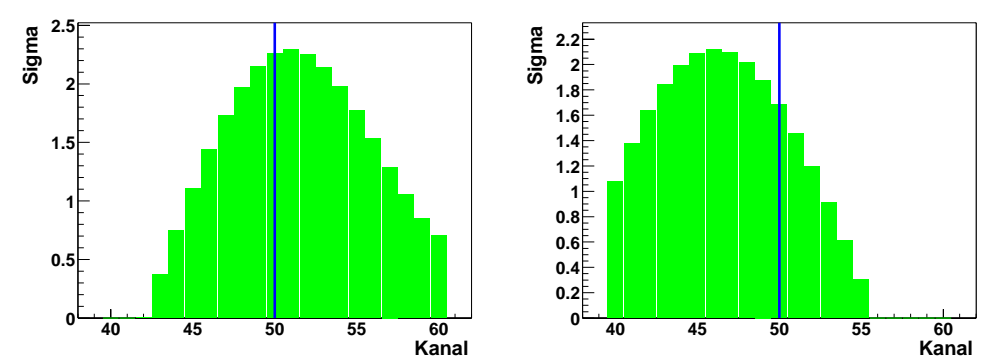

Figure 11: Two spectra with a Poisson-distributed background and a Gaussian line with 15 events centered in channel 50 (with a width (standard-deviation) of 4.0 channels) created with different random numbers. Shown is the result of the peak-scanning of the spectra. In the left picture the maximum of the probability corresponds well with the expected value (black line) whereas in the right picture a larger deviation is found. When a channel corresponds to $0.36 \mathrm{keV}$ the deviation in the right picture is $\sim 1.44 \mathrm{keV}$ (from [36 $\left.{ }^{1}, 1\right]$

Also simulated was the cosmic muon flux measured in the Gran Sasso, on the measured spectrum. To give a feeling for the quality of the simulation, Fig. '1] ${ }_{1}^{2}$ shows the simulated 

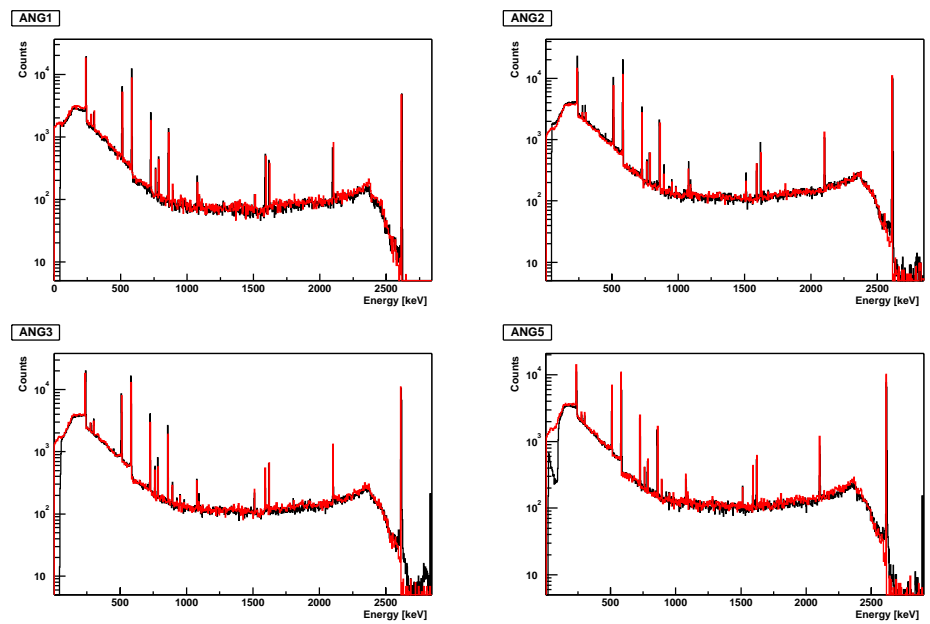

Figure 12: Comparison of the measured data (black line, November 1995 to April 2002) and simulated spectrum (red line) for the detectors Nrs. $1,2,3$ and 5 for a ${ }^{232} \mathrm{Th}$ source spectrum. The agreement of simulation and measurement is excellent (from [35is, 1127$]$ ).

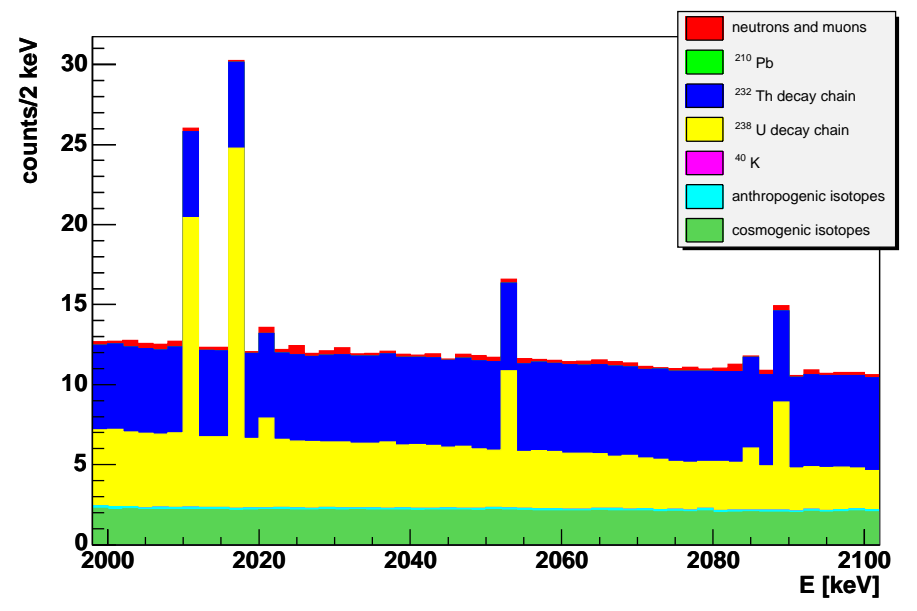

Figure 13: Simulated background of the HEIDELBERG-MOSCOW experiment in the energy range from 2000 to $2100 \mathrm{keV}$ with all known background components, for the period 20 November

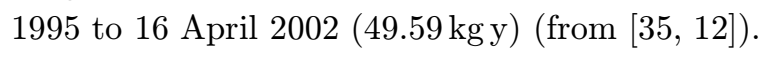

and the measured spectra for a ${ }^{228} \mathrm{Th}$ source spectrum for as example one of our five detectors. The agreement is excellent.

The simulation of the background of the experiment reproduces a 11 lines observed in the sum spectrum of the five detectors, in the energy range between threshold (around

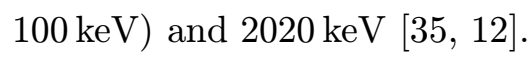

Fig. 1 in shows the simulated background in the range $2000-2100 \mathrm{keV}$ with all $\mathrm{k} \mathrm{n} \mathrm{o} \mathrm{w} \mathrm{n}$ background components. The black histogram corresponds to the measured data in the period 20.11.1995 - 16.4.2002 (49.59 kg y).

The background around $Q_{\beta \beta}$ is according to the simulations $\mathrm{fl}$ a t, the only expected 
lines come from ${ }^{214} \mathrm{Bi}$ (from the ${ }^{238} \mathrm{U}$ natural decay chain) at 2010.89, 2016.7, 2021.6, 2052.94, 2085.1 and $2089.7 \mathrm{keV}$. Lines from cosmogenically produced ${ }^{56} \mathrm{Co}$ (at $2034.76 \mathrm{keV}$ and $2041.16 \mathrm{keV}$ ), half-life 77.3 days, are not expected since the first 200 days of measurement of each detector are not used in the data analysis. Also the potential contribution from decays of ${ }^{77} \mathrm{Ge},{ }^{66} \mathrm{Ga}$, or ${ }^{228} \mathrm{Ac}$, should not lead to signals visible in our measured spectrum near the signal at $Q_{\beta \beta}$. For details we refer to [1] $\left.{ }_{1}^{1} \overline{2}\right]$.

\section{Proofs and disproofs}

The result described in section 2.1 has been questioned in some papers [Aalseth et al, hep-ex/0202018, and in Mod. Phys. Lett. A 17 1475-1478; Feruglio et al., Nucl. Phys. B 637 (2002) 345; Zdesenko et al., Phys. Lett. B 546 (2002) 206], and Kirpichnikov, talk at Meeting of Physical Section of Russian Academy of Sciences, Moscow, December 2, 2002, (and priv. communication, Dec. 3, 2002). We think that we have shown in a convincing way that these claims against our results are incorrect in various ways. In particular the estimates of the intensities of the ${ }^{214} \mathrm{~B} i$ lines in the first two papers do not take into account the effect of true coincidence summing, which can lead to drastic underestimation of the intensities. A correct estimate would also require a Monte Carlo simulation of our setup, which has not been performed in the above papers.

The paper by Zdesenko et al. starts from an arbitrary assumption, namely that there are lines in the spectrum at best only at 2010 and $2053 \mathrm{keV}$. This contradicts to the experimental result, according to which there are further lines in the spectrum (see Fig. $\overline{\underline{3}}_{1}$ in the present paper). For example they could have easily deduced from the intensity of the $2204 \mathrm{keV}$ Bi line in the measured spectrum that a line at $2053 \mathrm{keV}$ is expected with an

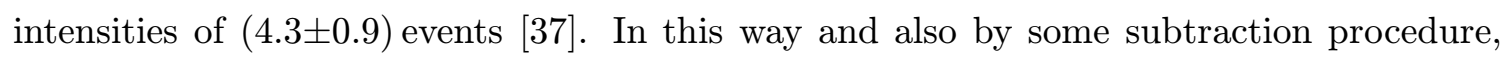
ignoring that the result of subtracting a Poisson distributed spectrum from a Poisson-

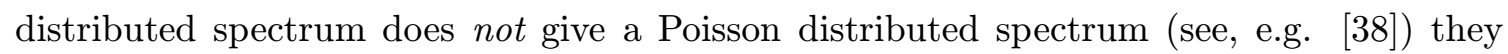
come to wrong conclusions.

Kirpichnikov states [i $4 \overline{1} \overline{1}]$ that from his analysis he clearly sees the $2039 \mathrm{keV}$ line in the full (not pulse-shape discriminated) spectrum with $>99 \%$ c.l. He claims that he does not see the signal in the pulse shape spectrum. One reason to see less intensity certainly is that in this case he averages for determination of the background over the full energy range without allowing for any lines. His result is in contradiction with the result we obtain under the same assumption of assuming just one line (at $\mathrm{Q}_{\beta \beta}$ ) and a continuous background (see Fig. 13 from [i]

All of these papers, when discussing the choice of the width of the search window, ignore the results of the statistical simulations - we present here, and have published in

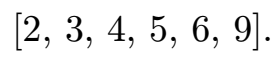

The strange effects found recently by the Kurchatov people [i62in in their rough analysis,

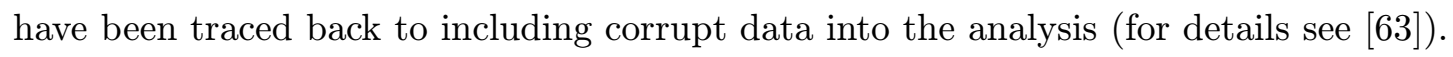




\section{Discussion of results}

\subsection{Half-life and effective neutrino mass}

We emphasize that we find in all analyses of our spectra a line at the value of $Q_{\beta \beta}$. We have shown that the signal at $\mathrm{Q}_{\beta \beta}$ should not originate from a background $\gamma$-line. On this basis we translate the observed number of events into half-lives for the neutrinoless double beta decay. We gave in Table 2 conservatively the values obtained with the Bayesian method. Also given in Table $\overline{2}_{i}$ are the effective neutrino masses $\langle m\rangle$ deduced using the

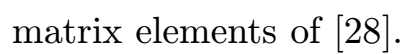

Table 2: Half-life for the neutrinoless decay mode and deduced effective neutrino mass from the HEIDELBERG-MOSCOW experiment (data 1990 - 2000).

\begin{tabular}{|c|c|c|c|c|}
\hline$k g y$ & Detectors & $\mathrm{T}_{1 / 2}^{0 \nu} \mathrm{y}$ & $\langle m\rangle \mathrm{eV}$ & C.L. \\
\hline 54.9813 & $1,2,3,4,5$ & $(0.80-35.07) \times 10^{25}$ & $(0.08-0.54)$ & $95 \%$ c.l. \\
& & $(1.04-3.46) \times 10^{25}$ & $(0.26-0.47)$ & $68 \%$ c.l. \\
& & $1.61 \times 10^{25}$ & 0.38 & Best Value \\
\hline 46.502 & $1,2,3,5$ & $(0.75-18.33) \times 10^{25}$ & $(0.11-0.56)$ & $95 \%$ c.l. \\
& & $(0.98-3.05) \times 10^{25}$ & $(0.28-0.49)$ & $68 \%$ c.l. \\
& & $1.50 \times 10^{25}$ & 0.39 & Best Value \\
\hline 28.053 & $2,3,5 \mathrm{SSE}$ & $(0.88-22.38) \times 10^{25}$ & $(0.10-0.51)$ & $90 \%$ c.l. \\
& & $(1.07-3.69) \times 10^{25}$ & $(0.25-0.47)$ & $68 \%$ c.l. \\
& & $1.61 \times 10^{25}$ & 0.38 & Best Value \\
\hline
\end{tabular}

We derive from the data taken with $46.502 \mathrm{~kg}$ y the half-life $\mathrm{T}_{1 / 2}^{0 \nu}=(0.8-18.3) \times 10^{25}$ y $(95 \%$ c.l.). The analysis of the other data sets, shown in Table particular importance is that we see the $0 \nu \beta \beta$ signal in the single site spectrum.

The result obtained is consistent with all other double beta experiments - which still reach in general by far less sensitivity. The most sensitive experiments following the HEIDELBERG-MOSCOW experiment are the geochemical ${ }^{128} \mathrm{Te}$ experiment with

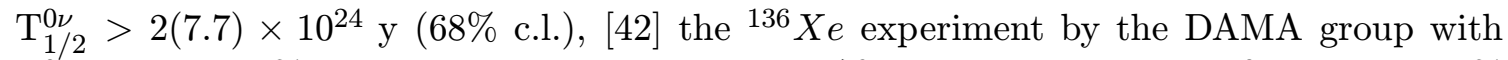
$\mathrm{T}_{1 / 2}^{0 \nu}>1.2 \times 10^{24}$ y $(90 \%$ c.l. $)$, a second enriched ${ }^{76} G e$ experiment with $\mathrm{T}_{1 / 2}^{0 \nu}>1.2 \times 10^{24}$

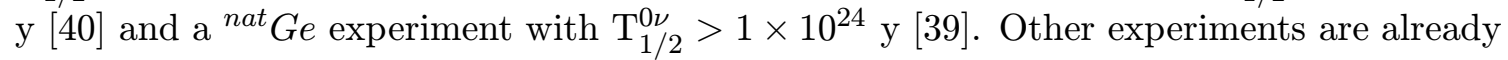
about a factor of 100 less sensitive concerning the $0 \nu \beta \beta$ half-life: the Gotthard TPC

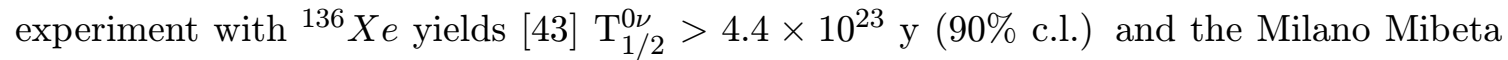
cryodetector experiment $\mathrm{T}_{1 / 2}^{0 \nu}>1.44 \times 10^{23}$ y (90\% c.l.).

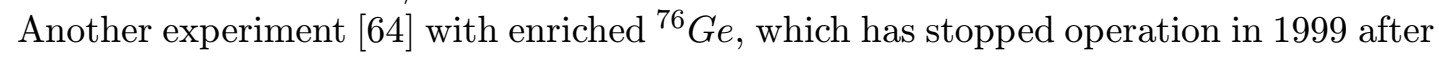
reaching a significance of $8.8 \mathrm{~kg}$ y, yields (if one believes their method of 'visual inspection' in their data analysis), in a conservative analysis, a limit of about $\mathrm{T}_{1 / 2}^{0 \nu}>5 \times 10^{24} \mathrm{y}$ (90\% c.l.). The ${ }^{128} \mathrm{Te}$ geochemical experiment yields $\left\langle m_{\nu}\right\rangle<1.1 \mathrm{eV}$ (68\% c.l.) [4늘] , the DAMA ${ }^{136} \mathrm{Xe}$ experiment $\left\langle m_{\nu}\right\rangle<(1.1-2.9) \mathrm{eV}$ and the ${ }^{130} \mathrm{Te}$ cryogenic experiment yields $\left\langle m_{\nu}\right\rangle<1.8 \mathrm{eV}$. 
Concluding we obtain, with $>95 \%$ probability, first evidence for the neutrinoless double beta decay mode. As a consequence, at this confidence level, lepton number is not conserved. Further the neutrino is a Majorana particle. If the $0 \nu \beta \beta$ amplitude is dominated by exchange of a massive neutrino the effective mass $\langle m\rangle$ is deduced (using the matrix elements of $[\overline{2} \overline{2} \bar{\nabla}])$ to be $\langle m\rangle=(0.11-0.56) \mathrm{eV}$ (95\% c.l.), with best value of $0.39 \mathrm{eV}$. Allowing conservatively for an uncertainty of the nuclear matrix elements of \pm $50 \%$ (for detailed discussions of the status of nuclear matrix elements we refer to and references therein) this range may widen to $\langle m\rangle=(0.05-0.84) \mathrm{eV}$ (95\% c.l.).

Assuming other mechanisms to dominate the $0 \nu \beta \beta$ decay amplitude, the result allows to set stringent limits on parameters of SUSY models, leptoquarks, compositeness, masses of heavy neutrinos, the right-handed $\mathrm{W}$ boson and possible violation of Lorentz invariance and equivalence principle in the neutrino sector. For a discussion and for references we

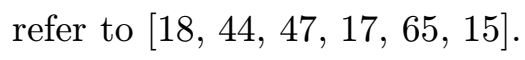

With the limit deduced for the effective neutrino mass, the HEIDELBERG-MOSCOW experiment excludes several of the neutrino mass scenarios allowed from present neutrino oscillation experiments (see Fig. 114in ) - allowing only for degenerate, and marginally still for inverse hierarchy mass scenarios [1] [1]

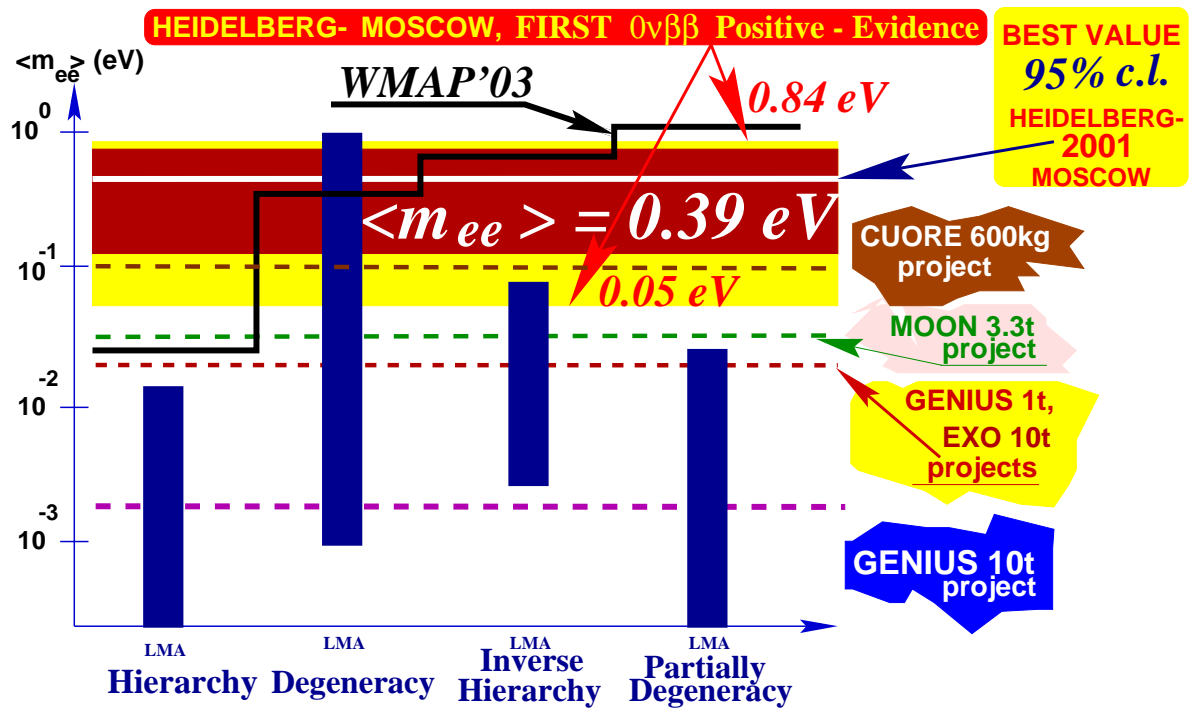

Figure 14: The impact of the evidence obtained for neutrinoless double beta decay (best value of the effective neutrino mass $\langle m\rangle=0.39 \mathrm{eV}, 95 \%$ confidence range $(0.05-0.84) \mathrm{eV}$ - allowing already for an uncertainty of the nuclear matrix element of a factor of $\pm 50 \%$ ) on possible neutrino mass schemes. The bars denote allowed ranges of $\langle m\rangle$ in different neutrino mass scenarios, still allowed by neutrino oscillation experiments (see $\left[1 \underline{4}_{0}^{1} i_{1}^{1} \underline{9}_{1}^{i}\right)$. Hierarchical models are excluded by the new $0 \nu \beta \beta$ decay result. Also shown is the exclusion line from WMAP, plotted for $\sum m_{\nu}<1.0 \mathrm{eV}$ [82i]. WMAP does not rule out any of the neutrino mass schemes. Further shown are the expected sensitivities for the future potential double beta experiments CUORE, MOON, EXO and the 1 ton and 10 ton project of GENIUS [1] $\left.\overline{1}_{1}^{1}, 1\right]$

The evidence for neutrinoless double beta decay has been supported by various recent experimental and theoretical results (see Table 1). Assuming the degenerate scenarios to 
be realized in nature we fix - according to the formulae derived in [i] $\overline{1} \overline{3}]$ - the common mass eigenvalue of the degenerate neutrinos to $\mathrm{m}=(0.05-3.4) \mathrm{eV}$. Part of the upper range is already excluded by tritium experiments, which give a limit of $\mathrm{m}<2.2-2.8 \mathrm{eV}$ (95\% c.l.) [i] $\left[\begin{array}{l}50 \\ 0\end{array}\right]$. The full range can only partly (down to $\sim 0.5 \mathrm{eV}$ ) be checked by future tritium decay experiments, but could be checked by some future $\beta \beta$ experiments (see next section). The deduced best value for the mass is consistent with expectations from experimental $\mu \rightarrow e \gamma$ branching limits in models assuming the generating mechanism for the neutrino mass to be also responsible for the recent indication for as anomalous magnetic moment of the muon [i]1]1]. It lies in a range of interest also for Z-burst models recently discussed as

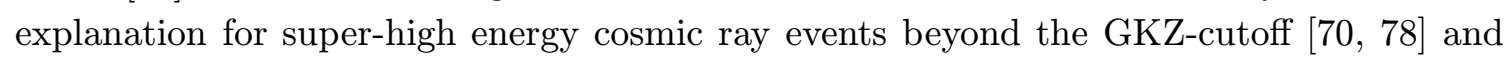
requiring neutrino masses in the range $(0.08-1.3) \mathrm{eV}$. A recent model with underlying $\mathrm{A}_{4}$ symmetry for the neutrino mixing matrix also leads to degenerate neutrino masses $>$

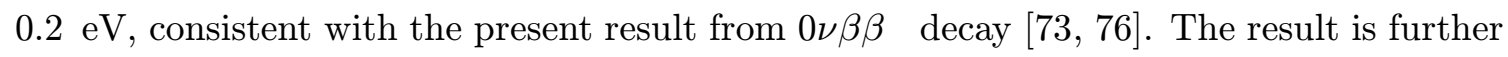

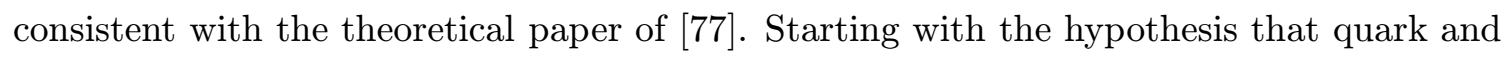
lepton mixing are identical at or near the GUT scale, Mohapatra et al. [7] $\left.\overline{7}_{1}\right]$ show that the large solar and atmospheric neutrino mixing angles can be understood purely as result of renormalization group evolution, if neutrino masses are quasi-degenerate (with same CP parity). The common Majorana neutrino mass then must be, in this model, larger than $0.1 \mathrm{eV}$.

For WMAP a limit on the total neutrino masses of

$$
m_{s}=\sum m_{i}<0.69 \mathrm{eV} \quad \text { at } 95 \% \text { c.l., }
$$

is given by the analysis of ref. [180 be very realistic. Another analysis shows that this limit on the total mass should be $[\overline{8} \overline{2}]$

$$
m_{s}=\sum m_{i}<1.0 \mathrm{eV} \quad \text { at } 95 \% \text { c.l. }
$$

The latter analysis also shows, that four generations of neutrinos are still allowed and in the case of four generations the limit on the total mass is increased to $1.38 \mathrm{eV}$. If there is a fourth neutrino with very small mass, then the limit on the total mass of the three neutrinos is even further weakened and there is essentially no constraint on the neutrino

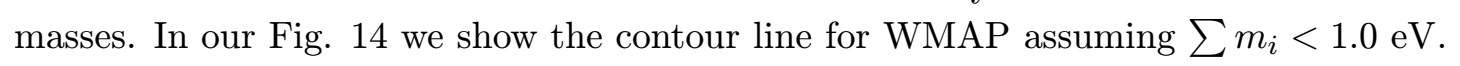

Comparison of the WMAP results with the effective mass from double beta decay rules

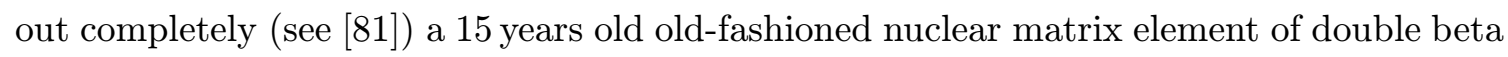
decay, used in a recent analysis of WMAP [i] element there was not included a realistic nucleon-nucleon interaction, which has been included by all other calculations of the nuclear matrix elements over the last 15 years.

As mentioned in section 1 the results from double beta decay and WMAP together may indicate $[\overline{2} \overline{0} \overline{0}]$ that the neutrino mass eigenvalues have indeed the same CP parity, as required by the model of [ī $\left.\bar{z}_{\overline{7}}\right]$.

The range of $\langle m\rangle$ fixed in this work is, already now, in the range to be explored by the

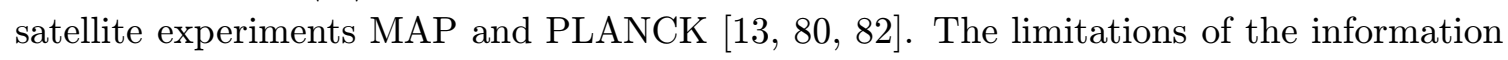

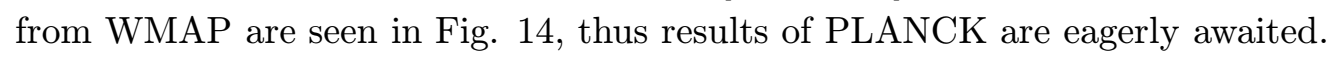

The neutrino mass deduced leads to $0.002 \geq \Omega_{\nu} h^{2} \leq 0.1$ and thus may allow neutrinos to still play an important role as hot dark matter in the Universe [i5 


\section{Future of $\beta \beta$ experiments - GENIUS and other proposals}

With the HEIDELBERG-MOSCOW experiment, the era of the small smart experiments is over. New approaches and considerably enlarged experiments (as discussed, e.g. in

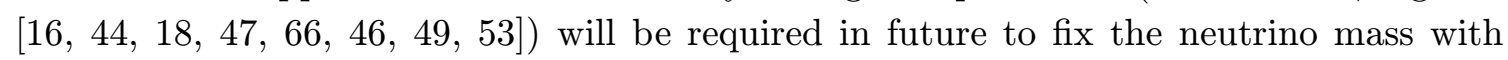
higher accuracy.

Since it was realized in the HEIDELBERG-MOSCOW experiment, that the remaining small background is coming from the material close to the detector (holder, copper cap, ...), elimination of any material close to the detector will be decisive. Experiments which do not take this into account, like, e.g. CUORE and MAJORANA will allow at best only rather limited steps in sensitivity. Furthermore there is the problem in cryodetectors that they cannot differentiate between a $\beta$ and a $\gamma$ signal, as this is possible in Ge experiments.

Another crucial point is the energy resolution, which can be optimized only in experiments using Germanium detectors, or, to some less extent, with bolometers. It will be difficult to probe evidence for this rare decay mode in experiments, which have to work as result of their limited resolution - with energy windows around $\mathrm{Q}_{\beta \beta}$ of several hundreds of $\mathrm{keV}$, such as NEMO III, EXO, CAMEO.

Another important point is the efficiency of a detector for detection of a $\beta \beta$ signal. For example, with $14 \%$ efficiency a potential future $100 \mathrm{~kg}{ }^{82} \mathrm{Se}$ NEMO experiment would be, because of its low efficiency, equivalent only to a $10 \mathrm{~kg}$ experiment (not talking about the energy resolution).

In the first proposal for a third generation double beta experiment, the GENIUS

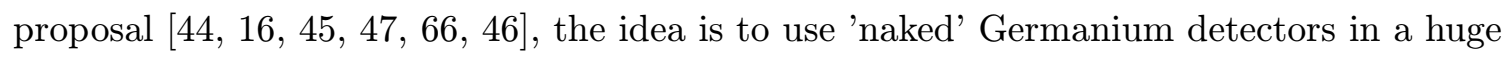
tank of liquid nitrogen. It seems to be at present the only proposal, which can fulfill both requirements mentioned above - to increase the detector mass and simultaneously reduce the background drastically. From our present knowledge (Dec. 2003) from the HEIDELBERG-MOSCOW experiment, it is questionable, whether GENIUS would be needed to confirm the results presented in this talk. Probably it would be preferable to perform an experiment with another isotope but fulfilling all requirements mentioned above. The other goals obtained by GENIUS, cold dark matter and solar neutrinos, also seem to have been solved by DAMA [i] $\left.\bar{g}_{1}\right]$, which has a $6.4 \sigma$ confidence level for observation of cold dark matter, and by Kamland [1] $\left.{ }_{1}^{4}\right]$, which seems to have proved (although still basing on a calculated reactor neutrino flux) that the LMA solution is the solution of the solar neutrino problem. GENIUS with ten tons of enriched ${ }^{76} \mathrm{Ge}$ might be of interest, however, to investigate whether the neutrino mass mechanism or another mechanism such as exchange of SUSY particles, (see, e.g. [1] $\left[\begin{array}{l}\overline{1} \\ \overline{8}\end{array}\right)$ is dominating the $0 \nu \beta \beta$ decay amplitude.

\section{GENIUS-TF}

As a first step to GENIUS, a small test facility, GENIUS-TF, is being installed in the Gran

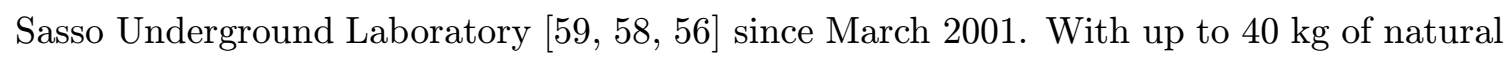
Ge detectors operated in liquid nitrogen, GENIUS-TF could test the DAMA seasonal modulation signature for dark matter [5흘. No other experiment running, like CDMS,

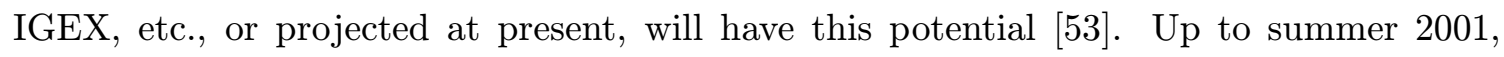


already six $2.5 \mathrm{~kg}$ Germanium detectors with an extreme low-level threshold of $\sim 500 \mathrm{eV}$ have been produced.

The original idea of GENIUS-TF was to prove the feasibility of some key constructional features of GENIUS, such as detector holder systems, achievement of very low thresholds of specially designed Ge detectors, long term stability of the new detector concept, reduction of possible noise from bubbling nitrogen, etc. Now the main idea is to look for seasonal modulations for dark matter.

After installation of the GENIUS-TF setup between halls A and B in Gran Sasso, opposite to the buildings of the HEIDELBERG-MOSCOW double beta decay experiment and of the DAMA experiment, the first four detectors have been installed in liquid nitrogen

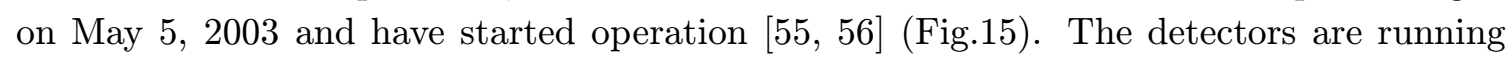
perfectly already 7 months now (for details see the talk by [5ㄴㄷㄴ.

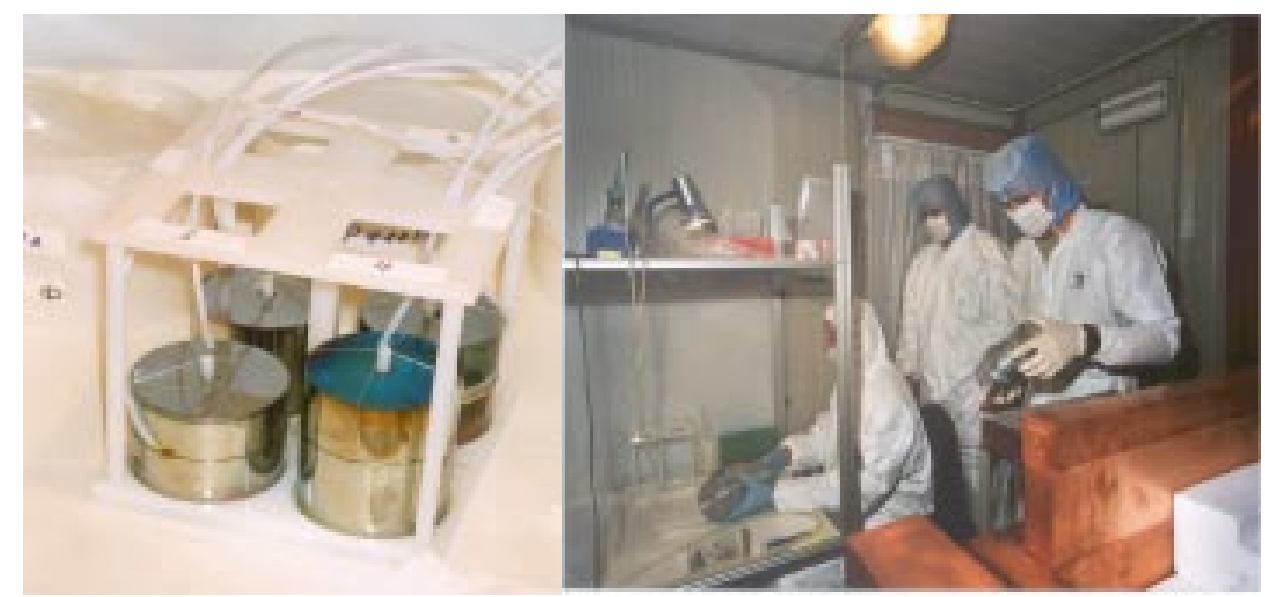

Figure 15: Left: The first four naked Ge detectors before installation into the GENIUS-TF setup. Right: Taking out the crystals from the transport dewars and fixing the electrical contacts in the clean room of the GENIUS-TF building - from left to right: Herbert Strecker, Hans Volker KlapdorKleingrothaus, Oleg Chkvorez.

This is the first time ever, that this novel technique for extreme background reduction, proposed in $\left[\overline{4} \overline{4} \overline{4}, \overline{4} \overline{4} \overline{5}_{1}, \overline{4} \overline{6}_{i}^{\prime}\right]$ in search for rare decays is tested under realistic background conditions in an underground laboratory.

\section{Conclusion}

The status of present double beta decay search has been discussed, and recent evidence for a non-vanishing Majorana neutrino mass obtained by the HEIDELBERG-MOSCOW experiment has been presented. Additional support for this evidence has been presented by showing consistency of the result - for the signal, a n d for the background - with other double beta decay experiments using non-enriched or enriched Germanium detectors (see also [6i signal (including those which at present cannot be attributed) are seen also in the other experiments. This is important for the correct treatment of the background. Furthermore, the sensitivity of the peak identification procedures has been demonstrated by extensive 
statistical simulations. It has been further shown by new extensive simulations of the expected background by GEANT4, that the background around $Q_{\beta \beta}$ should be flat, and that no known gamma line is expected at the energy of $Q_{\beta \beta}$ (see [1 $\left.\left.122^{2}\right]\right)$. The $2039 \mathrm{keV}$ signal is seen o $\mathrm{n} \mathrm{l} y$ in the HEIDELBERG-MOSCOW experiment, which has a by far larger statistics than all other double beta experiments.

The importance of this first evidence for violation of lepton number and of the Majorana nature of neutrinos is obvious. It requires beyond Standard Model Physics on one side, and may open a new era in space-time structure [i $\overline{6} \overline{8}]$. It has been discussed that the Majorana nature of the neutrino tells us that spacetime does realize a construct that is central to construction of supersymmetric theories.

Future projects to improve the present accuracy of the effective neutrino mass have been briefly discussed. The most sensitive of them and perhaps at the same time most realistic one, is the GENIUS project. GENIUS is the only of the new projects which simultaneously has a huge potential for cold dark matter search, and for real-time detection

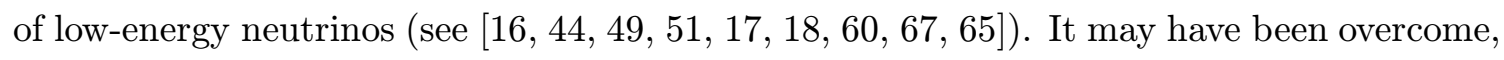
however, by the historical development.

With the successful start of operation of GENIUS-TF with the first four naked Ge

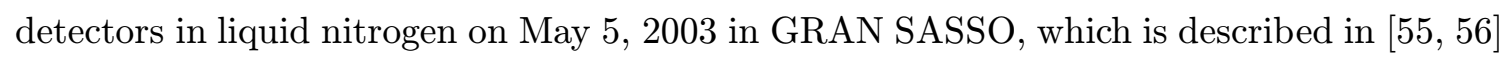
(see also the contribution of I.V. Krivosheina to these Proceedings) a historical step has been achieved of a novel technique and into a new domain of background reduction in underground physics in the search for rare events.

\section{References}

[1] H.V. Klapdor-Kleingrothaus et al. Mod. Phys. Lett. A 16 (2001) 2409 - 2420.

[2] H.V. Klapdor-Kleingrothaus, A. Dietz, I.V. Krivosheina, Part. and Nucl. 110 (2002) 57 - 79.

[3] H.V. Klapdor-Kleingrothaus, hep-ph/0205228, in Proc. of DARK2002, Cape Town, South Africa, February 4 - 9, 2002, eds. by H.V. Klapdor-Kleingrothaus and R.D. Viollier, Springer (2002) $404-411$.

[4] H.V. Klapdor-Kleingrothaus, hep-ph/0302248, Proc.DARK2002, Cape Town, South Africa, February 4 - 9, 2002, eds. by H.V. Klapdor-Kleingrothaus and R.D. Viollier, Springer (2002) $367-403$.

[5] H.V. Klapdor-Kleingrothaus, A. Dietz and I.V. Krivosheina, Foundations of Physics 31 (2002) 1181-1223 and Corr., 2003: http://www.mpi-hd.mpg.de/non_acc/main_results.html.

[6] H.V. Klapdor-Kleingrothaus, A. Dietz, I.V. Krivosheina, Ch. Dörr, C. Tomei, Phys. Lett. B 578 (2004) 54-62 and hep-ph/0312171.

[7] H.V. Klapdor-Kleingrothaus et al., (HEIDELBERG-MOSCOW Col.), Eur. Phys. J. A 12 (2001) 147, Proc. of "Third Internat. Conference on Dark Matter in Astro- and Particle Physics", DARK2000, ed. H.V. Klapdor-Kleingrothaus, Springer (2001) 520 - 533.

[8] H.V. Klapdor-Kleingrothaus, hep-ph/0205228, and in Proc. of DARK2002, Cape Town, South Africa, February 4 - 9, 2002, eds. by H.V. Klapdor-Kleingrothaus and R.D. Viollier, Springer, Heidelberg (2002) $404-411$. 
[9] H.V. Klapdor-Kleingrothaus, hep-ph/0303217 and in Proc. of "Neutrinos and Implications for Phys. Beyond the SM", Stony Brook, 11-13 Oct. 2002, World Scientific (2003) pp. 367-382.

[10] H.V. Klapdor-Kleingrothaus, A. Dietz, I.V. Krivosheina, Ch. Dörr, C. Tomei, Nuclear Instruments and Methods in Physics Research 510 A (2003) 281-289 and hep-ph/0308275.

[11] H.V. Klapdor-Kleingrothaus, O. Chkvorez, I. V. Krivosheina, C. Tomei, Nuclear Instruments and Methods in Physics Research 511 A (2003) 335-340 and hep-ph/0309157.

[12] Ch. Dörr and H.V. Klapdor-Kleingrothaus, Nuclear Instruments and Methods in Physics Research 513 A (2003) 596-621.

[13] H.V. Klapdor-Kleingrothaus, H. Päs and A.Yu. Smirnov, Phys. Rev. D 63 (2001) 073005 and hep-ph/0003219.

[14] H.V. Klapdor-Kleingrothaus and U. Sarkar, Mod. Phys. Lett. A 16 (2001) 2469 - 2482.

[15] H V Klapdor-Kleingrothaus, Special issue: Neutrinos, 2003, Proc. Indian Natl. Sci. Acad., hep-ph/0302237.

[16] H.V. Klapdor-Kleingrothaus, Int. J. Mod. Phys. A 13 (1998) 3953.

[17] H.V. Klapdor-Kleingrothaus, Springer Tracts in Modern Physics, 163 (2000) 69 - 104, Springer-Verlag, Heidelberg, Germany (2000).

[18] H.V. Klapdor-Kleingrothaus, "60 Years of Double Beta Decay - From Nuclear Physics to Beyond the Standard Model", World Scientific, Singapore (2001) 1281 p.

[19] H.V. Klapdor-Kleingrothaus and U. Sarkar, hep-ph/0304032, and Mod. Phys. Lett. A 18 (2003) 2243-2254.

[20] H.V. Klapdor-Kleingrothaus, to be publ. 2004, and in Proc. of Third International Conference on Particle Physics Beyond the Standard Model, BEYOND02, Oulu, Finland, 2-7 June 2002, ed. by H.V. Klapdor-Kleingrothaus, IOP, Bristol 2003, 215 - 240.

[21] M. Hirsch, H.V. Klapdor-Kleingrothaus and S.G. Kovalenko, Phys. Lett. B 398 (1997) 311, Phys. Lett. B 403 (1997) 291, and Phys. Rev. D 57 (1998) 1947.

[22] M. Hirsch, H.V. Klapdor-Kleingrothaus and S.G. Kovalenko, Phys. Rev. Lett. 75 (1995) 17, and Phys. Rev. D 53 (1996) 1329, and Phys. Lett. B 372 (1996) 181; and G. Bhattacharya, H.V. Klapdor-Kleingrothaus and H. Päs Phys. Lett. B 463 (1997) 77.

[23] J. Hellmig and H.V. Klapdor-Kleingrothaus, NIM A 455 (2000) 638-644.

[24] J. Hellmig, F. Petry and H.V. Klapdor-Kleingrothaus, Patent DE19721323A.

[25] B. Majorovits and H.V. Klapdor-Kleingrothaus. Eur. Phys. J. A 6 (1999) 463.

[26] CERN number generators (see e.g. http://root.cern.ch/root/html/TRandom.html)

[27] D.E Groom et al., Particle Data Group, Eur. Phys. J. C 15 (2000) 1.

[28] A. Staudt, K. Muto and H.V. Klapdor-Kleingrothaus, Eur. Lett. 13 (1990) 31.

[29] R.B. Firestone and V.S. Shirley, Table of Isotopes, 8th Ed., John W.\%S, N.Y. (1998).

[30] G. Gilmore et al."Practical Gamma-Ray Spectr.", Wiley and Sons (1995).

[31] G. Douysset et al., Phys. Rev. Lett. 86 (2001) 4259 - 4262.

[32] J.G. Hykawy et al., Phys. Rev. Lett. 67 (1991) 1708. 
[33] G. Audi, A.H. Wapstra, Nucl. Phys. A 595 (1995) 409-480.

[34] R.J. Ellis et al., Nucl. Phys. A 435 (1985) 34-42.

[35] Ch. Dörr, Diplomarbeit (2002), Univ. of Heidelberg, unpubl.

[36] A. Dietz, Dissertation, University of Heidelberg, 2003.

[37] K. Ya. Gromov, priv. communication, 2003.

[38] M.D. Hannam, W.J. Thompson, Nucl. Instr. Meth. A 431 (1999) 239-251.

[39] D. Caldwell, J. Phys. G 17, S137-S144 (1991).

[40] I.V. Kirpichnikov et al. Mod. Phys. Lett. A 5 (1990) 1299 - 1306. Preprint ITEP, 1991, Moscow 91-91.

[41] I.V. Kirpichnikov, priv. communication, Dec. 3, 2002.

[42] O. Manuel et al., in Proc. Intern. Conf. Nuclear Beta Decays and the Neutrino, eds. T. Kotani et al., World Scientific (1986) 71, J. Phys. G: Nucl. Part. Phys. 17 (1991) S221-S229; T. Bernatovicz et al. Phys. Rev. Lett. 69 (1992) 2341 - 2344.

[43] R. Lüscher et al., Phys. Lett. (1998) 407.

[44] H.V. Klapdor-Kleingrothaus in Proc. of BEYOND'97, First International Conference on Particle Physics Beyond the Standard Model, Castle Ringberg, Germany, 8-14 June 1997, ed. by H.V. Klapdor-Kleingrothaus and H. Päs, IOP Bristol (1998) 485-531.

[45] H.V. Klapdor-Kleingrothaus, J. Hellmig and M. Hirsch, J. Phys. G 24 (1998) 483 - 516.

[46] H.V. Klapdor-Kleingrothaus et al. MPI-Report MPI-H-V26-1999, hep-ph/9910205, in Proc. of the 2nd Int. Conf. on Particle Physics Beyond the Standard Model BEYOND'99, Castle Ringberg, Germany, 6-12 June 1999, eds. H.V. Klapdor-Kleingrothaus and I.V. Krivosheina, IOP Bristol (2000) 915-1014.

[47] H.V. Klapdor-Kleingrothaus, in Proc. of NEUTRINO 98, Takayama, Japan, 4-9 Jun 1998, (eds) Y. Suzuki et al. Nucl. Phys. Proc. Suppl. 77 (1999) 357 - 368.

[48] HeIDELBERG-MOSCOW Coll. (M. Günther et al.), Phys. Rev. D 55 (1997) 54.

[49] H.V. Klapdor-Kleingrothaus, Nucl. Phys. B 100 (2001) 309 - 313.

[50] J. Bonn et al., Nucl. Phys. B 91 (2001) 273 - 279.

[51] V.A. Bednyakov and H.V. Klapdor-Kleingrothaus, hep-ph/0011233 and Phys. Rev. D 63 (2001) 095005.

[52] H.V. Klapdor-Kleingrothaus, in Proc. of the Int. Symposium on Advances in Nuclear Physics, eds.: D. Poenaru and S. Stoica, World Scientific, Singapore (2000) 123-129.

[53] H.V. Klapdor-Kleingrothaus, Int. J. Mod. Phys. A 17 (2002) 3421 -3431, and in Proc. of Intern Conf. LP01, WS 2002, Rome, Italy, July 2001.

[54] H.V. Klapdor-Kleingrothaus and I.V. Krivosheina, in Proc. of International Workshop on Astroparticle and High Energy Physics, Valencia, Spain, September 2003.

[55] H.V. Klapdor-Kleingrothaus, CERN Courier, 2003. 
[56] H.V. Klapdor-Kleingrothaus, O. Chkvorez, I.V. Krivosheina, H. Strecker, C. Tomei, Nuclear Instruments and Methods in Physics Reserach A 511 (2003) 341 - 346 and hep-ph/0309170, and H.V. Klapdor-Kleingrothaus and I.V. Krivosheina, in Proc. of Beyond the Desert 2002, BEYOND02, Oulu, Finland, June 2002, IOP 2003, ed. H.V. Klapdor-Kleingrothaus.

[57] C. Tomei, A. Dietz, I. Krivosheina, H.V. Klapdor-Kleingrothaus, Nuclear Instruments and Methods in Physics Reserach A 508 (2003) 343-352, and hep-ph/0306257.

[58] T. Kihm, V. F. Bobrakov and H.V. Klapdor-Kleingrothaus NIM A498 (2003) 334-339 and hep-ph/0302236.

[59] H.V. Klapdor-Kleingrothaus et al., hep-ph/0103082, NIM A 481 (2002) 149-159.

[60] H.V. Klapdor-Kleingrothaus and I.V. Krivosheina, in Proc. of "Forum of Physics", Zacatecas, Mexico, 11-13 May, 2002, eds. D.V. Ahluwalia and M. Kirchbach, Found. Phys. 33 (2003) 831-837.

[61] C. Weinheimer, in Appec meeting, Karlsrhue, 16-18 September 2003, http://www-ik.fzk.de/\%7ekatrin/atw/talks.html, and J. Bonn et al., Nucl. Phys. Proc. Suppl. 110 (2002) 395-397.

[62] A.M. Bakalyarov et al. (Moscow grop of HEIDELBERG-MOSCOW experiment), hep-ex/0309016.

[63] H.V. Klapdor-Kleingrothaus et al., in preparation.

[64] C.E. Aalseth et al. (IGEX Collaboration), Yad. Fiz. 63, No 7 (2000) 1299 - 1302; Phys. Rev. D 65 (2002) 092007.

[65] H.V. Klapdor-Kleingrothaus, Part. and Nucl., Lett. 104 (2001) 20-39, hep-ph/0102319.

[66] H.V. Klapdor-Kleingrothaus, hep-ph/0103074 and in Proc. NOON 2000, World Scientific, Singapore (2001) 219-234.

[67] H.V. Klapdor-Kleingrothaus, Proc. LowNu2, World Scientific, Singapore (2001) 116-131 and hep-ph/0104028.

[68] D.V. Ahluwalia in Proc. of Beyond the Desert 2002, BEYOND02, Oulu, Finland, June 2002, IOP 2003, ed. H.V. Klapdor-Kleingrothaus, 143-160; D.V. Ahluwalia, M. Kirchbach, Phys. Lett. B 529 (2002) 124.

[69] R. Bernabei et al., Riv. Nuovo Cim. 26 (2003) 1-73.

[70] D. Fargion et al., in Proc. of DARK2000, Heidelberg, Germany, July 10-15, 2000, Ed. H.V. Klapdor-Kleingrothaus, Springer, (2001) 455 -468 and in Proc. of Beyond the Desert 2002, BEYOND02, Oulu, Finland, June 2002, IOP 2003, ed. H.V. Klapdor-Kleingrothaus.

[71] E. Ma and M. Raidal, Phys. Rev. Lett. 87 (2001) 011802; Erratum-ibid. 87 (2001) 159901.

[72] Y. Uehara, Phys. Lett. B 537 (2002) 256-260 and hep-ph/0201277.

[73] E. Ma in Proc. of Intern. Conf. on Physics Beyond the Standard Model: Beyond the Desert 02, BEYOND'02, Oulu, Finland, 2-7 Jun. 2002, IOP, Bristol (2003) 95 - 106, ed. H.V. Klapdor-Kleingrothaus.

[74] KamLAND Coll., Phys. Rev. Lett. 90 (2003) 021802 and hep-ex/0212021.

[75] G. L. Fogli et al., Phys. Rev. D 67 (2003) 073002 and hep-ph/0212127.

[76] K. S. Babu, E. Ma and J.W.F. Valle (2002) hep-ph/0206292. 
[77] R. N. Mohapatra, M. K. Parida and G. Rajasekaran, (2003) hep-ph/0301234.

[78] Z. Fodor, S. D. Katz and A. Ringwald, Phys. Rev. Lett. 88 (2002) 171101; Z. Fodor et al., JHEP (2002) 0206:046, or hep-ph/0203198, and in Proc. of Intern. Conf. on Physics Beyond the Standard Model: Beyond the Desert 02, BEYOND'02, Oulu, Finland, 2-7 Jun 2002, IOP, Bristol, 2003, ed. H V Klapdor-Kleingrothaus and hep-ph/0210123.

[79] J.E. Ruhl et al., astro-ph/0212229.

[80] D.N. Spergel et al., Astrophys. J. Suppl. 148 (2003) 175 and astro-ph/0302209.

[81] A. Pierce and H. Murayama, hep-ph/0302131.

[82] S. Hannestad, JCAP 0305 (2003) 004 and astro-ph/0303076.

[83] P. Vogel in PDG (ed. K Hagiwara et al.) Phys. Rev. (2002) D 66010001.

[84] S.W. Allen, R.W. Schmidt, S.L. Bridle, Astron. Astrophys. 412 (2003) 35-44 and astro-ph/0306386.

[85] A. Blanchard, M. Douspis, M. Rowan-Robinson, S. Sarkar, astro-ph/0304237. 